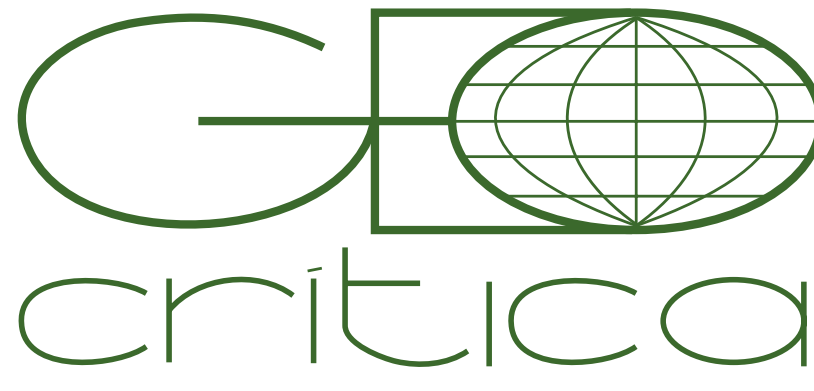

\section{Scripta Nova}

Revista Electrónica de Geografía y Ciencias Sociales Universidad de Barcelona

ISSN: 1138-97

Vol. XXI. Núm. 559

1 de marzo de 2017

\title{
CONTAMINACIÓN DEL AIRE, MORTALIDAD CARDIOVASCULAR Y GRUPOS VULNERABLES EN MADRID: UN ESTUDIO EXPLORA- TORIO DESDE LA PERSPECTIVA DE LA JUSTICIA AMBIENTAL
}

\author{
Prieto-Flores, María-Eugenia'; \\ Moreno Jiménez, Antonioza; \\ Gómez-Barroso, Diana3; \\ Cañada Torrecilla, Rosa ${ }^{2 b}$; \\ Martínez Suárez, Pedro²c.
}

\author{
'Departamento de Geografía, Universidad Nacional de Educación a Distancia. \\ meprietof@geo.uned.es \\ 2Departamento de Geografía, Universidad Autónoma de Madrid. \\ (a) antonio.moreno@uam.es (b) rosa.canada@uam.es (c) pedromarts@hotmail.com \\ ${ }^{3}$ CIBER Epidemiología y Salud Pública. Centro Nacional de Epidemiología, Instituto de Salud Carlos III. \\ dgomez@externos.isciii.es
}

Recibido: 13 de diciembre de 2015. Aceptado: 11 de octubre de 2016.

Los autores agradecen a María Jesús Vidal Domínguez la preparación de la cartografía digital de las secciones censales para el AUP de Madrid y la obtención y elaboración de datos de los grupos sociodemográficos por secciones censales. Este trabajo ha sido realizado a través los Proyectos de Investigación "Contaminación atmosférica urbana y justicia ambiental: metodología de evaluación y estudio de casos con Sistemas de Información Geográfica" (Ref. CSO201126177) y "Polución del aire, poblaciones vulnerables y salud: análisis de injusticias ambientales basados en geotecnologías" (Ref. CSO201455535-R), financiados por el Ministerio de Economía y Competitividad de España.

\begin{abstract}
Resumen. Contaminación del aire, mortalidad cardiovascular y grupos vulnerables en Madrid: un estudio exploratorio desde la perspectiva de la justicia ambiental

La perspectiva de la justicia ambiental está contribuyendo a desvelar la interacción entre amenazas ambientales, condiciones de salud y grupos vulnerables. En este artículo se estudian de manera integrada para la ciudad de Madrid los patrones espaciales de ciertos grupos sociodemográficos vulnerables, de contaminación atmosférica por partículas y de mortalidad cardiovascular (controlando el efecto de la edad). Para ello se ha articulado ex novo una metodología de análisis espacial que incluye técnicas estadísticas y un sistema de información geográfica, para identificar posibles desigualdades socio-espaciales.
\end{abstract}

Palabras clave: Justicia ambiental, grupos sociodemográficos vulnerables, contaminación atmosférica, mortalidad cardiovascular, sistemas de información geográfica, Madrid.

\begin{abstract}
Air pollution, cardiovascular mortality and vulnerable groups in Madrid: an exploratory study from the perspective of environmental justice.

The environmental justice perspective is contributing to unravel the interaction among environmental threats, health conditions and vulnerable groups. The spatial patterns in Madrid City of some vulnerable socio-demographic groups, particulate air pollution and cardiovascular mortality are studied in an integrated manner in this paper. With this aim, an ex novo methodology of spatial analysis has been designed, involving statistical techniques and a geographic information system, to identify possible socio-spatial inequalities.
\end{abstract}

Key words: Environmental justice, vulnerable socio-demographic groups, particulate air pollution, cardiovascular mortality, geographic information systems, Madrid. 
El estudio de la relación entre los desequilibrios ambientales, las disparidades socioeconómicas y las desigualdades en salud ha sido abordado desde diversas áreas del conocimiento tales como la salud pública, la epidemiología y la geografía de la salud. Una perspectiva de investigación con un alto contenido teórico, metodológico y práctico, centrado en estas dimensiones interrelacionadas, es la de la justicia ambiental, que de una manera integradora dirige su atención a los aspectos físicos y sociales de la salud asociados a la distribución de los beneficios y los riesgos ambientales entre las poblaciones ${ }^{1}$. En este sentido, una de las definiciones de justicia ambiental de más larga tradición, aportada por la Agencia de Protección Ambiental de Estados Unidos en 1998, destaca que todas las personas deben tener un trato justo independientemente de cuestiones tales como su origen, ingresos y nivel educativo, con respecto al desarrollo y aplicación de políticas ambientales y que ninguna población debería estar obligada a soportar en mayor medida los efectos de los problemas medioambientales en la salud.

Diversos aportes evidencian que las personas con un menor nivel socioeconómico se encuentran más expuestas a las amenazas ambientales, incluyendo la polución del aire y del agua, la contaminación acústica, y la proximidad a zonas de desechos peligrosos, entre otros ${ }^{2}$. En este contexto, numerosos trabajos han demostrado que las causas de la injusticia ambiental se encuentran en la desigualdad económica, la exclusión social y la discriminación racial ${ }^{3}$.

La revisión de la literatura presentada por Walker ${ }^{4}$ aporta una aproximación a los temas de estudio más recientes en justicia ambiental en relación con cada una de sus dimensiones. En la dimensión social se destacan, por ejemplo, cuestiones relacionadas con diferencias de género, población mayor, discapacidad, clases sociales, derechos ambientales de poblaciones originarias y responsabilidades hacia las futuras generaciones. En cuanto a la dimensión ambiental, se ha abordado en los últimos años la polución atmosférica, los residuos, la calidad del agua, el ruido, los espacios verdes y el cambio climático, entre otros muchos problemas. En relación con el significado de justicia, tal y como lo demuestra el mismo autor, hay diversos puntos de vista, sin embargo, todas las definiciones coinciden en incluir nociones de distribución de recursos y de riesgos.

Otro componente de gran importancia es el espacial, pues el campo de la justicia ambiental se ocupa del entorno definido como el lugar donde vivimos, trabajamos, jugamos y vamos al colegio, entre otras actividades cotidianas, además de

\footnotetext{
1 Institute of Medicine Committee on Environmental Justice 1999

2 Brulle y Pellow 2006

3 Mohai, Pellow y Roberts 2009

4 Walker 2012
} 
considerar el entorno natural ${ }^{5}$. Así mismo, tal y como indican Walker y Bulkeley ${ }^{6}$, el concepto de justicia ambiental siempre ha tenido un contenido profundamente geográfico en la investigación de patrones espaciales y sociales de los riesgos ambientales, observándose desigualdades en el territorio, en relación con los diferentes grupos sociales.

Según la Organización Mundial de la Salud (OMS), los problemas ambientales como la contaminación atmosférica contribuyen a acentuar las desigualdades sociales en la salud, y a pesar de los esfuerzos por incrementar la calidad del aire en los países desarrollados, la polución del mismo sigue siendo un tema central de investigación con el objetivo de mejorar la salud pública de las poblaciones? ${ }^{7}$ Tal como lo recoge este informe, habría una exposición y una susceptibilidad diferencial de los grupos de población más vulnerables. La primera alude a que estos grupos se encuentran expuestos con mayor frecuencia a la contaminación atmosférica, mientras que la segunda indica que dichos grupos pueden ser más susceptibles a los efectos de la polución en la salud, debido a una mayor prevalencia de enfermedades pre-existentes en dicha población.

Entre los contaminantes del aire, en el contexto europeo se han investigado las partículas en suspensión ( $\mathrm{PM}_{2,5}$ y PM10), el dióxido de nitrógeno $\left(\mathrm{NO}_{2}\right)$, el ozono $\left(\mathrm{O}_{3}\right)$ y el dióxido de azufre $\left(\mathrm{SO}_{2}\right)$, entre otros, con relación a distintos indicadores socioeconómicos, especialmente en el Reino Unido ${ }^{7}$, observándose que una peor calidad del aire afecta a zonas de mayor privación socioeconómica. A su vez, la contaminación atmosférica ha demostrado efectos negativos en la salud, aumentando el riesgo de morbilidad y mortalidad cardiovascular y respiratoria, entre otras afecciones ${ }^{8,9}$.

Si bien el número de artículos científicos desarrollados en Europa en esta línea va en aumento, todavía es escaso y aún queda mucho por comprender ${ }^{10}$. Tal y como indican estos autores, algunos de estos trabajos demuestran que los grupos de población más pobres se encuentran más expuestos a la contaminación del aire, si bien en otros casos se han observado poblaciones más ricas con una mayor exposición, por residir en zonas de viviendas de clase alta con un elevado tráfico viario. No obstante, los distintos resultados coinciden en evidenciar que los grupos socioeconómicos más desfavorecidos son los que experimentan en mayor medida los efectos nocivos de la contaminación atmosférica por su mayor vulnerabilidad.

La complejidad de las relaciones entre la dimensión social, ambiental y de salud ha dado lugar a la aplicación de diversos métodos y herramientas de análisis, entre los cuales los Sistemas de Información Geográfica (SIG) contribuyen a profundizar en la comprensión de las relaciones espaciales entre estas tres dimensiones. De este

5 Bullard 2007

6 Walker y Bulkeley 2006

7 World Health Organization 2010

8 Jerrett et al. 2005

9 World Health Organization 2013

10 Deguen y Zmirou-Navier 2010 
modo, Maantay ${ }^{11}$, en su estudio sobre el papel de los SIG en la valoración de la salud y la equidad ambiental, se refiere a las siguientes utilidades: la representación espacial de las tasas de morbilidad ayuda a identificar patrones no observados anteriormente; los SIG permiten combinar características de salud de nivel individual con datos agregados de exposición a factores ambientales, en unidades geográficas como secciones censales; los SIG se pueden utilizar para seleccionar estudios de caso y para elaborar y confirmar hipótesis relacionadas con riesgos ambientales; la información espacial aportada mediante los SIG contribuye a la toma de decisiones para mejorar las intervenciones en materia de salud pública.

En su estudio sobre el estado de la cuestión de la justicia ambiental, Arriaga Legarda y Pardo Buendía ${ }^{12}$ señalan que los trabajos empíricos en este campo, en el contexto español, son todavía incipientes. Tal y como indican las autoras, algunas de las contribuciones más importantes han sido realizadas especialmente por geógrafos ${ }^{13,14,15,16,17}$. En estos trabajos se analiza, mediante técnicas estadísticas y SIG, la exposición a distintas amenazas ambientales, tales como la contaminación sonora y la polución del aire por $\mathrm{NO}_{2}, \mathrm{PM}_{10}$ y $\mathrm{SO}_{2}$, identificándose desequilibrios socioespaciales e inequidades en su distribución, que afectan en mayor medida a algunos grupos socioeconómicos. Por su parte, el Observatorio de la Sostenibilidad en España ha aportado estudios de diagnóstico y propuestas sobre los efectos de la calidad del aire en la salud, donde se incluye el impacto de la contaminación atmosférica en grupos vulnerables, a partir de los estudios que vienen realizando investigadores de áreas científicas de salud y medio ambiente en España ${ }^{18}$.

Con el presente trabajo se pretende avanzar en los estudios de justicia ambiental en España, integrando la triple dimensión ambiental, social y de la salud en una investigación empírica en el municipio de Madrid. El componente ambiental se analizará mediante la contaminación atmosférica por partículas en suspensión con un tamaño inferior o igual a 10 micras, conocido como $\mathrm{PM}_{10}$. El social será estudiado a través de distintos grupos sociodemográficos vulnerables considerando criterios de edad (personas mayores de 80 años), nivel de estudios y ámbito de origen de la población inmigrante de países menos desarrollados. La dimensión de salud, finalmente, se examinará a través de la mortalidad cardiovascular, una de las primeras causas de fallecimiento en países desarrollados.

Estudios previos han evidenciado la relación entre polución del aire y mortalidad por causas cardiovasculares ${ }^{19,20}$. Las concentraciones de partículas en suspen-

11 Maantay 2002

12 Arriaga Legarda y Pardo Buendía 2011

13 Bosque Sendra, Díaz Castillo y Díaz Muñoz 2001-2002

14 Cañada Torrecilla 2007

15 Cañada Torrecilla, Vidal Domínguez y Moreno Jiménez 2011

16 Moreno Jiménez 2007

17 Moreno Jiménez 2013

18 Fundación Mapfre 2013

19 Routledge y Ayres 2005

20 Wong et al. 2008 
sión muestran asociaciones significativas no solo con muertes cardiovasculares registradas en los certificados médicos de defunción, sino también con infartos de miocardio clínicamente confirmados y con fibrilación ventricular ${ }^{21}$. También se ha observado una mayor exposición a la contaminación atmosférica por partículas en población con mayor privación social en lo referente a ingresos, empleo, educación, salud, vivienda y acceso a servicios ${ }^{22,23}$.

En este contexto, el objetivo planteado en este trabajo estriba en determinar si existe en el municipio de Madrid una situación de inequidad expresada en el patrón espacial de $\mathrm{PM}_{10}$, mortalidad cardiovascular y grupos sociodemográficos de población. Así, se busca conocer cómo se distribuyen estos grupos en función, a la vez, de las zonas de mayor o menor contaminación y de mayor o menor mortalidad por enfermedades cardiovasculares, para deducir si determinados colectivos se ven más afectados por esos riesgos ambientales de tipo atmosférico. Procede resaltar, como rasgo singular de nuestro estudio, que se aborda la interacción de tres fenómenos o dimensiones, el ambiental, el sociodemográfico y el de la salud, frente a muchos otros, dentro de esta línea de trabajo, en los que solo contemplan dos de ellos. Esa particularidad conlleva una dificultad superior, cuyo abordaje ha exigido un planteamiento metodológico nuevo.

En el apartado siguiente se presentan y justifican las fuentes de datos y los métodos utilizados, para luego exponer los resultados alcanzados. Tras un apartado de discusión se proponen las conclusiones.

\section{Métodos y fuentes de información}

La complejidad analítica de los objetivos planteados ha precisado recurrir a fuentes variadas de datos, a un conjunto de decisiones metodológicas y a una pluralidad de técnicas cuantitativas y tecnologías que se expondrán a continuación.

\section{Fuentes y preparación de datos}

En primer lugar, establecer una adecuada delimitación del ámbito de estudio en la ciudad de Madrid resulta una cuestión previa importante. Dentro del espacio urbanizado existen zonas de muy desigual presencia humana y, además, la movilidad habitual de la población impide, por ahora, conocer con exactitud de su exposición al ambiente atmosférico donde se halla en cada momento. Pese a las evidentes limitaciones, la alternativa más frecuente ha sido referir la población a su domicilio habitual y considerar que las condiciones ambientales de ese entorno son las que representarían mejor dicha exposición potencial al ambiente atmosférico. Así se ha hecho en este estudio, para lo cual se ha definido el concepto de "área urbana poblada" (AUP), como aquella en la que hay una notable presencia habitual de residentes, tomando en cuenta también su entorno cercano. De manera operativa se incluyeron

21 Dockery 2001

22 Jerrett et al. 2001

23 Pye, Stedman, Adams y King 2001 
en la ciudad las zonas de uso residencial, comercial, ocio, etc. y se excluyeron las áreas extensas de uso industrial, transporte (e. g. aeropuertos), agrícola, ganadero, forestal, etc. donde la densidad de población era baja o exigua (véase figura l A). La delimitación de dicha zona se apoyó en la interpretación de imágenes aéreas recientes (Plan Nacional de Ortofotografía Aérea del Instituto Geográfico Nacional) y de cartografía de usos del suelo (Corine LandCover, 2006), apoyada en SIG ${ }^{24}$.

En segundo lugar, los datos de contaminación del aire por $\mathrm{PM}_{10}$ proceden de los organismos locales y autonómicos competentes (Ayuntamiento y Comunidad de Madrid). Se han utilizado estaciones sitas en el interior del municipio de Madrid y otras próximas situadas en municipios limítrofes, para mejorar la cobertura del muestreo espacial. La variable ambiental seleccionada para este estudio ha sido la concentración media anual de partículas en suspensión de un tamaño igual o inferior a $10 \mu \mathrm{m}, \mathrm{PM}_{10^{\prime}}$ del año 2010 en $\mu \mathrm{g} / \mathrm{m}^{3}$; estas son uno de los contaminantes con los que está relacionada la mortalidad cardiovascular. La media anual se obtiene a partir de datos horarios que previamente han sido validados por el Servicio de Protección de la Atmósfera. No ha sido necesario recurrir al relleno de lagunas. Como es sabido, las partículas (aerosoles) son emitidas por una gran variedad de fuentes, en función de las cuales varían sus propiedades físicas y su composición química. Pueden ser primarias, vertidas directamente a la atmósfera desde su fuente de emisión, o secundarias, formadas a partir de contaminantes primarios. La principal fuente en el ámbito urbano es el tráfico rodado, que engloba tanto emisiones directas de la combustión, como las procedentes por la resuspensión de materiales procedentes del firme de la calzada, como consecuencia de la abrasión mecánica de vehículos, frenos, ruedas, etc. Una parte importante también se debe a emisiones derivadas de obras de construcción, demolición, etc. Su concentración depende de la dinámica atmosférica, que influye en su dispersión, estancamiento o en su transporte, como se ha puesto de manifiesto en numerosos estudios sobre Barcelona o el Mediterráneo Occidental ${ }^{25,26,27 .}$

Puesto que los datos de dichas estaciones conforman una muestra espacial, y como para la evaluación de la equidad ambiental se precisa conocer el nivel de polución en todos los lugares de la ciudad, se avistó acometer una estimación de los mismos. La estimación de la contaminación se puede realizar desde diferentes enfoques, la elección de uno u otro está condicionada por la disponibilidad de datos: modelos de dispersión de contaminantes ${ }^{28,29,30,31}$, el uso de la regresión ${ }^{8,32,33}$, o las

25 Querol et al. 2003

26 Rodríguez, Querol, Alastuey y Plana 2002

27 Viana et al. 2005

28 Daly y Zannetti 2007

29 Fan, Lam y Yu 2012

30 Maroko 2012

31 Wang, Van Den Bosch y Kuffer 2008

32 Brauer et al. 2003

33 Su et al. 2010 
técnicas de interpolación espacial ${ }^{15,16,22,34,35,36,37}$. En nuestro caso se ha optado por la utilización de estas últimas.

La metodología aplicada está detallada en Cañada Torrecilla et al. ${ }^{38}$. Se diseñó un procedimiento con varias etapas. En la primera se realizó un análisis exploratorio de los datos muestrales de contaminación. En la segunda se aplicó un análisis estructural de los mismos basado en el semivariograma, con el objeto de identificar las direcciones de máxima y mínima continuidad de la tendencia espacial. En caso de detectar anisotropía espacial se definiría una elipse cuyos ejes mayor y menor reflejarían adecuadamente la misma. En caso de no detectar anisotropía se optaría por el modelo isotrópico, en el que sólo se tendría en cuenta la variación de los valores en función de la distancia. En la tercera etapa del análisis se ensayó la aplicación de dos métodos de interpolación, la media ponderada por el inverso de la distancia (IDW) y el Kriging ordinario ${ }^{14,37,39}$. El objetivo siguiente fue confrontar sus resultados y dirimir la aceptabilidad de cada uno de ellos, adoptando a la vez criterios estadísticos convencionales, como la bondad de ajuste, y otros geográficos, más cualitativos, como la coherencia con el marco territorial y la estructura urbana local. La aplicación de estas técnicas se realizó con ArcGIS (Geostatistical Analyst).

En el estudio de la contaminación por partículas el método que proporcionó un mejor resultado fue el Kriging anisotrópico, por lograr unos menores errores en la predicción: el mean error (ME), media de los errores próxima a 0, el root mean square error (RMSE), error cuadrático medio más bajo y el root mean square standarized error (RMSSE) próximo a l (cuadrol). Ello implicó seleccionar los puntos muestrales para interpolar en cada lugar adoptando una vecindad de forma elíptica con el tamaño, la orientación, el número de sectores y de puntos próximos más idóneos, que condujesen además a unas menores diferencias entre los valores observados y pronosticados por el modelo (cuadro 2).

Una vez obtenida la capa estimada de media anual de $\mathrm{PM}_{10}$ se convirtió a raster con una resolución de 50 metros y se recortó para ajustarla al AUP. Seguidamente, y para imputar valores de contaminación a las unidades de análisis (las secciones censales) se realizó un tratamiento con ArcGIS (Zonal statistics as a table) que calculó para cada una de ellas la media de los niveles de polución en los píxeles incluidos en dicha sección.

34 Buzzelli y Jerrett 2004

35 De Mesnard 2013

36 Li y Heap 2011

37 Wong, Yuan y Perlin 2004

38 Cañada Torrecilla, Moreno Jiménez y González Lorenzo 2014

39 Krivoruchko 2011 


\begin{tabular}{|c|c|c|}
\hline Modelo & Parámetros & Ciudad de Madrid \\
\hline \multirow{4}{*}{$\begin{array}{c}\text { Kriging } \\
\text { anisotrópico }\end{array}$} & $\mathrm{N}^{\mathrm{o}}$ vecinos & $7 / 3$ \\
\cline { 2 - 3 } & Búsqueda de vecindad & $\begin{array}{c}\text { Elipse, 4 partes, 45 } \\
9000 / 5000\end{array}$ \\
\cline { 2 - 3 } & $\begin{array}{c}\text { Ángulo de rotación de la } \\
\text { elipse }\end{array}$ & $35^{\circ}$ \\
\hline \multirow{3}{*}{$\begin{array}{c}\text { Bondad de } \\
\text { ajuste }\end{array}$} & Media de los errores (ME) & $-0,04$ \\
\cline { 2 - 3 } & $\begin{array}{c}\text { Error cuadrático medio } \\
\text { (RMSE) }\end{array}$ & 3,58 \\
\cline { 2 - 3 } & RMSSE & 0,98 \\
\hline
\end{tabular}

Cuadro 1. Parámetros utilizados en la interpolación de las PM10 y bondad de ajuste del modelo Fuente: Elaboración propia

\begin{tabular}{|c|c|c|}
\hline \multirow{2}{*}{$\begin{array}{c}\text { Estadísticos } \\
\text { Descriptivos }\end{array}$} & \multicolumn{2}{|c|}{ Madrid } \\
\cline { 2 - 3 } & $\begin{array}{c}\text { Datos } \\
\text { observados }\end{array}$ & $\begin{array}{c}\text { Datos } \\
\text { estimados }\end{array}$ \\
\hline Media & 22,85 & 22,13 \\
\hline $\begin{array}{c}\text { Desviación } \\
\text { típica }\end{array}$ & 4,36 & 2,26 \\
\hline Máximo & 34 & 26,96 \\
\hline Mínimo & 15 & 16,86 \\
\hline Amplitud & 19 & 10,10 \\
\hline $\mathrm{n}$ & 20 & \\
\hline
\end{tabular}

Cuadro 2. Estadísticos descriptivos de los valores observados y pronosticados por el Kriging Fuente: Elaboración propia

Una particularidad de nuestro análisis estriba en que requiere adoptar límites para valorar la aceptación o no de la concentración del contaminante en la atmósfera. La aplicación a tal fin del criterio de la OMS o de la Unión Europea/España conduce a situaciones desiguales y parcialmente contradictorias: en general en Madrid no se supera el valor de $40 \mu \mathrm{g} / \mathrm{m}^{3}$, que es el nivel medio anual admisible por la legislación española y de la Unión Europea (UE), por lo que de utilizarse este umbral no habría problema ambiental; si se adopta el umbral más exigente de $20 \mu \mathrm{g} / \mathrm{m}^{3}$ (valor objetivo fijado por la OMS) en Madrid aflorarían zonas por encima y por debajo de ese valor. Considerando que la polución es un problema a reducir en el tiempo hasta niveles aceptables se adoptó para Madrid el umbral más exigente, que es el de $20 \mu \mathrm{g} / \mathrm{m}^{3}$. Una exposición prolongada a esta contaminación contribuye al riesgo de desarrollar enfermedades cardiovasculares, respiratorias y cáncer de pulmón 9, 40 . Estudios realizados en la UE indican que la esperanza de vida se puede reducir en 8,6 meses $^{41}$.

40 Querol et al. 2006

41 Gurjar, Molina y Ojha 2010 
Los datos de mortalidad se obtuvieron del Instituto de Estadística de la Comunidad de Madrid, por sexo, edad y sección censal de residencia de las personas fallecidas durante el año 2010. Las causas básicas de defunción analizadas aquí fueron las correspondientes al capítulo IX de la CIE 10: mortalidad por enfermedades del sistema circulatorio (International Statistical Classification of Diseases and Related Health Problems). Como indicador se eligió la Razón de Mortalidad Estandarizada (RME), para cuyo cálculo se utilizó la población del Padrón Municipal de Habitantes del mismo año del INE, estratificada por sexo y cuatro grupos de edad (0-14,15-64, 65-79 y 80 y más años ) a nivel de sección censal para el AUP del municipio de Madrid.

Para calcular dicha RME ajustada por edad se estimaron los casos esperados para cada unidad geográfica ( secciones censales del AUP), tomando como referencia la tasa global del municipio de mortalidad para las enfermedades cardiovasculares. Una vez obtenidos los casos esperados se calculó la RME para cada sección censal, teniendo como numerador los casos observados de mortalidad por enfermedad cardiovascular y como denominador los casos esperados para cada unidad. La RME mide, pues, el riesgo relativo de una sección censal respecto a un ámbito mayor de referencia, en este caso la ciudad de Madrid ${ }^{42}$. Aquellas secciones censales en las que la RME es mayor que 1 indican que el riesgo en ellas está por encima de la tasa del municipio.

La determinación de los grupos sociodemográficos a examinar desde la óptica de las posibles inequidades ambientales constituye otra decisión importante. En la bibliografía sobre justicia ambiental se ha considerado una amplia variedad de criterios para seleccionar grupos de población que pueden considerarse prioritarios a estos efectos por resultar más vulnerables o desfavorecidos, así como diversos indicadores socioeconómicos, de renta, pobreza, privación, etc. algunos de ellos compuestos $^{43}$.

En este estudio, se ha priorizado el criterio de vulnerabilidad humana (que en parte suele asociarse al de privación), seleccionándose un acotado número de grupos de población, cuyos datos estaban disponibles en el Padrón Municipal de Habitantes, para pequeñas unidades espaciales (secciones censales), que poseen entre 1000-2000 habitantes. La cartografía digital de las secciones censales procede del Instituto Nacional de Estadística 2010 y fue editada para ajustarse al AUP. El conjunto final de grupos elegidos para este estudio fueron los siguientes:

Según la edad, las personas de edad avanzada ( $\geq 80$ años) resultan, por sus rasgos biológicos, más sensibles a las agresiones ambientales ${ }^{44}$ y a sufrir las enfermedades cardiovasculares ${ }^{45}$.

Los inmigrantes extranjeros por razones económicas, provenientes de países menos desarrollados que la UE y que se insertan en la base de la pirámide laboral,

42 Gómez-Barroso, et al. 2015

43 Moreno Jiménez et al. 2016

44 Landrigan, Rauh y Galvez 2010

45 Maeso Martínez et al. 2006 
conforman otro grupo que, aunque dispar según la procedencia, suele sufrir más privaciones y unas condiciones sociales más adversas. Para dilucidar las posibles relaciones dentro de este heterogéneo colectivo se distinguieron varios subgrupos según procedencia, dada la notoria tendencia a la coalescencia y segregación que suelen exhibir en el interior de las ciudades. En concreto se han retenido como subgrupos los procedentes de América Latina, los africanos (procedentes sobre todo del Magreb y del África subsahariana), los asiáticos (predominantemente de China) y los europeos provenientes de países menos desarrollados (sobre todo Rumanía, Bulgaria, Polonia, Rusia y Ucrania).

Finalmente, y desde un criterio de estatus socioeconómico, se ha integrado la población de 30-59 años con bajo nivel de estudios (hasta enseñanza primaria incompleta). Para comparación y contraste experimental se incluyó también la población de 30-59 años con alto nivel de instrucción (universitaria o similar).

Los efectivos de población total y de tales grupos por secciones censales fueron imputados exclusivamente al espacio dentro del AUP. Esta información fue integrada en un SIG (ArcGIS) y manejada tanto bajo el modelo vectorial, como el raster (capas de densidad de población por píxeles de $50 \mathrm{~m}$ de resolución, i.e. 0,25 ha), para fines analíticos y de cartografía ${ }^{24}$.

\section{La metodología para evaluar la equidad ambiental}

El planteamiento del problema realizado en este trabajo involucra tres componentes, cuya interconexión se trata de desvelar: los grupos vulnerables, la contaminación por $\mathrm{PM}_{10}$ y la mortalidad por causas cardiovasculares. Antes de exponer los métodos aplicados conviene aclarar sucintamente la lógica subyacente al análisis, para que aquéllos queden justificados y comprendidos. La hipótesis general y escueta radica en presumir, en primer lugar, que una elevada concentración media de ese contaminante atmosférico es un factor negativo para la salud de los residentes en una zona. Así mismo, una elevada RME por causas cardiovasculares en una zona expresa una realidad desfavorable, resultante de procesos complejos y causas múltiples, entre las cuales cabe presumir también una atmósfera contaminada por partículas.

La exploración de la relación entre esos dos fenómenos, es decir, si las zonas con mayor (menor) concentración de $\mathrm{PM}_{10}$ tienden a exhibir mayores (menores) ratios de mortalidad estandarizada se realizó mediante la aplicación de técnicas estadísticas de correlación y ajustes de tendencia (lineales y no lineales), el coeficiente y test de independencia de la $\chi^{2}$, tras una dicotomización de las dos variables, según los criterios que más adelante se explican, y el test U de Mann-Whitney, para dos muestras independientes, que conlleva dicotomizar solo una de las variables (la RME). Así mismo se utilizó el gráfico de barras de error para examinar diferencias significativas entre grupos de secciones, tras separarlas mediante un criterio (variable) dicotomizado.

En todo caso, la hipótesis de (in)justicia ambiental, tal como aquí proponemos, 
pone el foco en averiguar si las condiciones adversas (o favorables) en los dos indicadores mencionados afectan proporcionalmente más (o menos) a los grupos vulnerables elegidos. Dado que la evaluación de tal justicia demanda una norma de comparación respecto a la cual establecer si un determinado grupo vulnerable aparece como perjudicado (o favorecido), se ha adoptado como referencia a la población total de la ciudad. Es decir, la incógnita a despejar aquí estriba en si un determinado grupo vulnerable está "expuesto" desproporcionadamente a unas condiciones ambientales y de mortalidad negativas (o no), esto es, alta polución y alta razón de mortalidad. Dado que no se dispone de medidas de exposición real a la contaminación aérea, ni de razones de mortalidad por grupos de población, lo que podemos indirectamente examinar es si cada uno de los grupos vulnerables reside proporcionalmente más en zonas donde la contaminación y la mortalidad son altas (o bajas). La sobrerrepresentación o sobre-abundancia de un grupo vulnerable concreto en tales ámbitos podría interpretarse como una penalización excesiva, y por tanto injusta, siempre en comparación con la población residente total en la ciudad.

El planteamiento adoptado parte, pues, de la definición de niveles elevados de contaminación y de mortalidad, de acuerdo con los parámetros expuestos anteriormente. Así, respecto a la polución por $\mathrm{PM}_{10}$ se utilizó el valor medio anual de $20 \mu \mathrm{g} /$ $\mathrm{m}^{3}$, y respecto a la razón de mortalidad se adoptó la unidad. En consecuencia, las secciones censales fueron sometidas a una doble clasificación: a) en primer lugar separando la categoría de las que superaban los $20 \mu \mathrm{g} / \mathrm{m}^{3}$ de media anual de $\mathrm{PM}_{10}$ (mayor contaminación) y, por otro, las que quedaban por debajo (menor contaminación); b) y en segundo lugar, las que excedían o estaban por debajo de 1 en la RME (mayor o menor riesgo de mortalidad cardiovascular, respectivamente). El cruce de ambas clasificaciones generó, pues, cuatro clases que se muestran en el cuadro 3 junto con la cifra de secciones censales de cada clase.

\begin{tabular}{|c|c|c|c|}
\hline \multicolumn{2}{|c|}{$\mathbf{P M}_{10}<\mathbf{2 0} \boldsymbol{\mu g} / \mathbf{m}^{\mathbf{3}}$} & $\mathbf{P M}_{10} \geq \mathbf{2 0} \boldsymbol{\mu g} / \mathbf{m}^{\mathbf{3}}$ \\
\hline RME-CV & RME-CV & RME-CV & RME-CV \\
$\leq 1$ & $>1$ & $\leq 1$ & $>1$ \\
\hline $102 \mathrm{sec}$. & $81 \mathrm{sec}$. & $1229 \mathrm{sec}$. & $988 \mathrm{sec}$. \\
\hline
\end{tabular}

Cuadro 3. Categorías analíticas según contaminación por $\mathrm{PM}_{10}$ y RME y distribución de las secciones censales

Fuente: Elaboración propia

Nota: RME-CV = Razón de mortalidad estandarizada cardiovascular

Seguidamente, se computaron las cifras de población (total y de los distintos grupos demográficos) en cada una de las cuatro clases establecidas, obteniendo las correspondientes distribuciones de frecuencias (con la población total y de cada grupo). Ello se ejecutó mediante selecciones temáticas ( $S Q L$ Select) en la base de datos temática de las secciones censales de ArcGIS.

Finalmente, la evaluación de la hipótesis de justicia ambiental se ha sustentado en dos técnicas estadísticas complementarias. 
Asumiendo que los grupos demográficos elegidos suponen una muestra de la población total se ha procedido, en primer lugar, a comprobar globalmente si la distribución de frecuencias de tales grupos en las cuatro categorías antedichas era similar a la de la población total de Madrid. A tal fin resulta apropiado el test $\chi^{2}$ de una muestra, perteneciente al tipo de la bondad de ajuste ${ }^{46,47}$, aplicado anteriormente ${ }^{48,49}$. Se trata de un conocido test de independencia mediante el cual se busca comprobar la hipótesis nula, $\mathrm{H}_{\mathrm{o}^{\prime}}$ de que la distribución de un grupo de población en las cuatro clases es idéntica a la que tiene el conjunto de la población residente. La hipótesis alternativa, $\mathrm{H}_{1}$, supone lo contrario, es decir, que son diferentes. Aceptar $\mathrm{H}_{0}$ implicaría que las condiciones de polución y la RME de dicho grupo demográfico son concordantes con las del universo de referencia y que, por tanto, no exhibe discriminación (injusticia). Si el test avalase $\mathrm{H}_{1}$ entonces sí que dicho grupo estaría expuesto a condiciones de contaminación por $\mathrm{PM}_{10}$ Y de RME diferentes. Lo que no sería factible dilucidar con tal test es si esas diferencias serían más favorables o más perjudiciales para dicho grupo, respecto a las de la población total.

Con el fin de dar respuesta a esta segunda cuestión se ha recurrido a una segunda herramienta que se centra en comparar las frecuencias relativas de cada grupo con las de la población total en cada una de las cuatro categorías separadamente. De esa forma es posible detectar en cuáles de tales categorías es significativa la diferencia y si ésta es por exceso o por defecto, es decir, si la proporción del grupo es mayor o menor que la de la población en cada clase. La técnica adoptada para ello ha sido el conocido test de diferencias entre proporciones (muestral y poblacional), basado en el estadístico de contraste $\mathrm{Z}^{50}$. Como es sabido, dicho estadístico arroja un valor tipificado cuya distribución es normal, la cual permite establecer la probabilidad de que una determinada diferencia de proporciones (i.e. el estadístico de contraste Z) ocurra por azar.

En nuestro caso, $\mathrm{H}_{\mathrm{o}}: \mathrm{p}=\pi_{\mathrm{o}} \mathrm{Y}$

$$
\mathrm{H}_{1}: \mathrm{p} \neq \pi_{\mathrm{o}^{\prime}}
$$

siendo $\mathrm{p}=$ la proporción del grupo en una clase dada y $\pi_{\mathrm{o}}=$ la proporción de la población en dicha clase. El contraste se ha realizado para cada una de las categorías o clases establecidas analíticamente y permite concluir, por tanto, sobre las divergencias de forma pormenorizada. Si aquella probabilidad ( $p$-valor) es muy baja cabe afirmar que la diferencia es estadísticamente significativa al nivel de confianza dado por dicho $p$-valor.

A la hora de interpretar los resultados y habida cuenta de las limitaciones en los datos de partida, se pondrá especial atención en analizar las categorías que com-

46 Ruiz-Maya, Martín Pliego, Montero y Uriz Tomé 1995

47 Siegel 1972

48 Moreno Jiménez 2010

49 Moreno Jiménez 2012

50 Hortelano 2000 
binan baja polución y baja RME, por un lado, y alta polución y alta RME, por otro, ya que, en caso de que la proporción de un grupo de población concreto excediese significativamente a la de la población total señalaría que tal grupo estaría "privilegiado" o "desfavorecido" respectivamente. En este último caso, se podría hablar además de injusticia ambiental.

Resta por añadir que los anteriores tratamientos estadísticos han sido realizados con el sistema NCSS (versión 9) y SPSS (versión 17).

En los apartados que siguen se presentarán, en primer lugar, los rasgos básicos y las distribuciones espaciales del indicador de contaminación ambiental $\left(\mathrm{PM}_{10}\right)$, de mortalidad cardiovascular (RME), así como de los grupos de población en la ciudad de Madrid. Seguidamente se expondrán los resultados de los análisis de las relaciones estadístico-espaciales (test estadísticos) entre los tres tipos de indicadores expuestos aquí.

\section{Análisis de resultados}

\section{La distribución intraurbana del contaminante $\mathbf{P M}_{10}$}

El mapa de la contaminación estimada del aire por esas partículas revela que los niveles más elevados se sitúan en la parte central y en zonas de la periferia SE y $S$ (figura 1), con valores superiores a $25 \mu \mathrm{g} / \mathrm{m}^{3}$, pudiendo relacionarse en el primer caso a las emisiones de tráfico y en el segundo a las partículas provenientes de suelos secos y desnudos de vegetación que abundan allí. Las zonas de menor polución conforman una banda perimetral discontinua que discurre desde el $\mathrm{N}$ hacia el W y SW, con cifras por debajo de $20 \mu \mathrm{g} / \mathrm{m}^{3}$.

La ciudad de Madrid no tendría riesgo de contaminación por $\mathrm{PM}_{10}$, considerando el umbral de $40 \mu \mathrm{g} / \mathrm{m}^{3}$ establecido por la legislación europea. Sin embargo, de acuerdo con el umbral fijado por la OMS (límite aplicado aquí), sí se aprecia desigualdades en la calidad del aire. Así, con ese umbral de $20 \mu \mathrm{g} / \mathrm{m}^{3}$, Madrid tendría el 77,3\% de la superficie del AUP como zona de riesgo.

\section{La distribución intraurbana de la mortalidad cardiovascular}

La RME ajustada por edad a nivel de sección censal muestra el patrón espacial de la mortalidad por enfermedades cardiovasculares en el AUP. El indicador posee una distribución de frecuencias con notable asimetría positiva, existiendo además unas pocas secciones anómalamente alejadas por la parte alta (cuadro 4). Los valores superiores a 1 indican que esa RME en la sección censal excede a la de referencia, la ciudad de Madrid, lo que equivale a decir que el riesgo de esa causa de muerte allí es más alto. El examen del mapa (figura 2) revela un patrón algo complejo y abigarrado. En no pocos ámbitos aflora la heterogeneidad, encontrándose secciones censales con valores por encima de 1 (más riesgo) vecinas de otras secciones con valores por debajo de 1 en las que el riesgo es menor (autocorrelación negativa). Con todo, se pueden entrever algunas tendencias espaciales. Para el caso de las secciones con alto 
RME (valores > 1) hay racimos de ellas en diversos lugares de distritos interiores y algunas grandes manchas en ciertas áreas periféricas (W, NE, SE y S). Adviértase que tales manchas periféricas son a menudo una sola sección, cuyo tamaño demográfico no es sin embargo muy diferente de otras secciones muy pequeñas del interior. Por su lado, las secciones con menor riesgo por esa causa de muerte (valores $<1$ ), aparte de en la trama urbana interior, exhiben una presencia destacada en la periferia, soe todo en el NW, SE y SW.

Figura 1. A) Niveles estimados de concentración media anual de $\mathrm{PM}_{10}$ en la atmósfera para Madrid en 2010; B) zona excediendo $20 \mu \mathrm{g} / \mathrm{m} 3$.

Fuente. Elaboración propia.

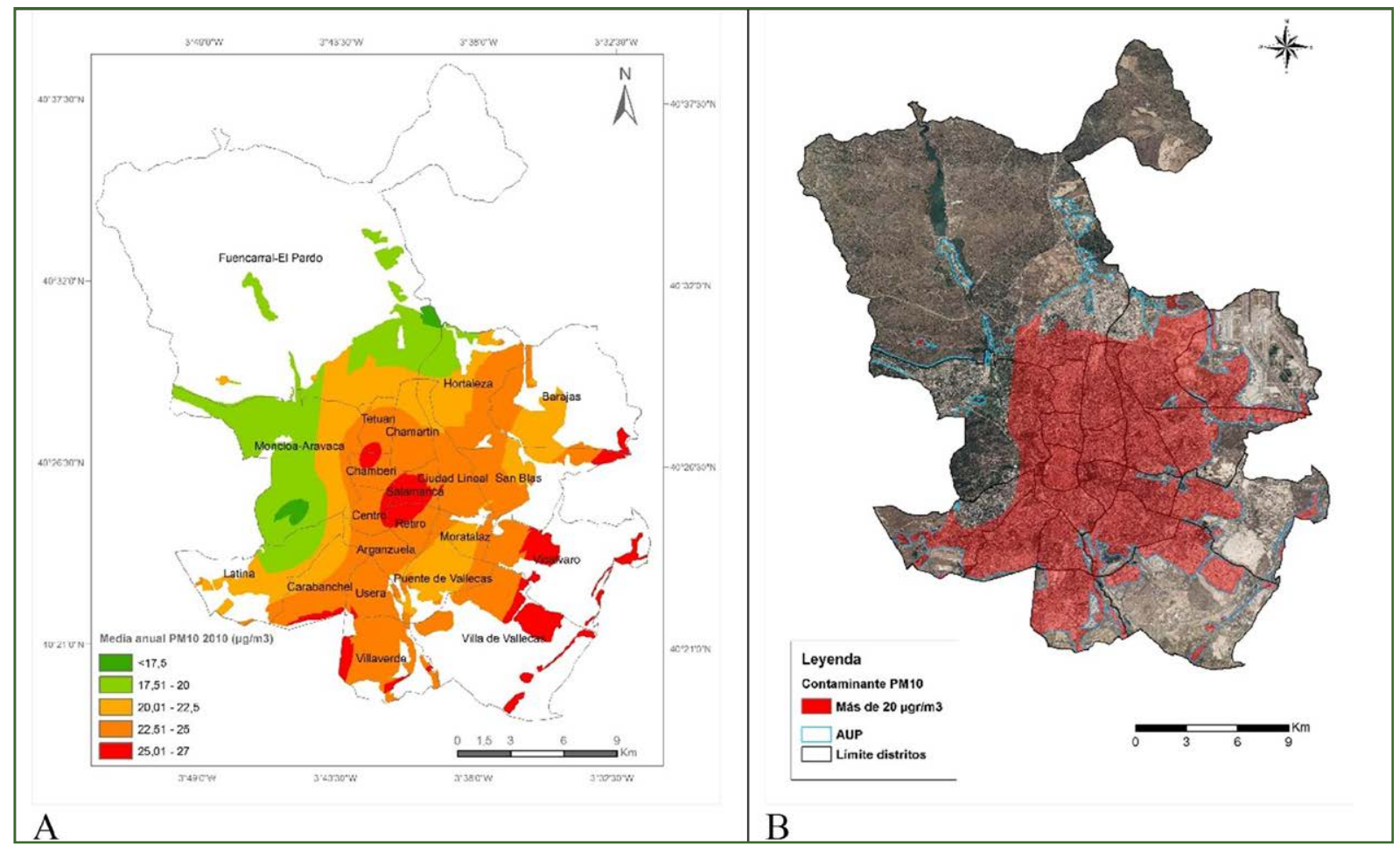




\begin{tabular}{|l|c|}
\hline PARÁMETRO & VALOR \\
\hline Media & 1,12 \\
\hline Mediana & 0,91 \\
\hline Desviación típica & 1,14 \\
\hline Mínimo & 0 \\
\hline Máximo & 20,67 \\
\hline Asimetría & 5,35 \\
(con error típico de 0,05) & \\
\hline
\end{tabular}

Cuadro 4. Rasgos del indicador RME cardiovascular por secciones censales

Fuente: Elaboración propia

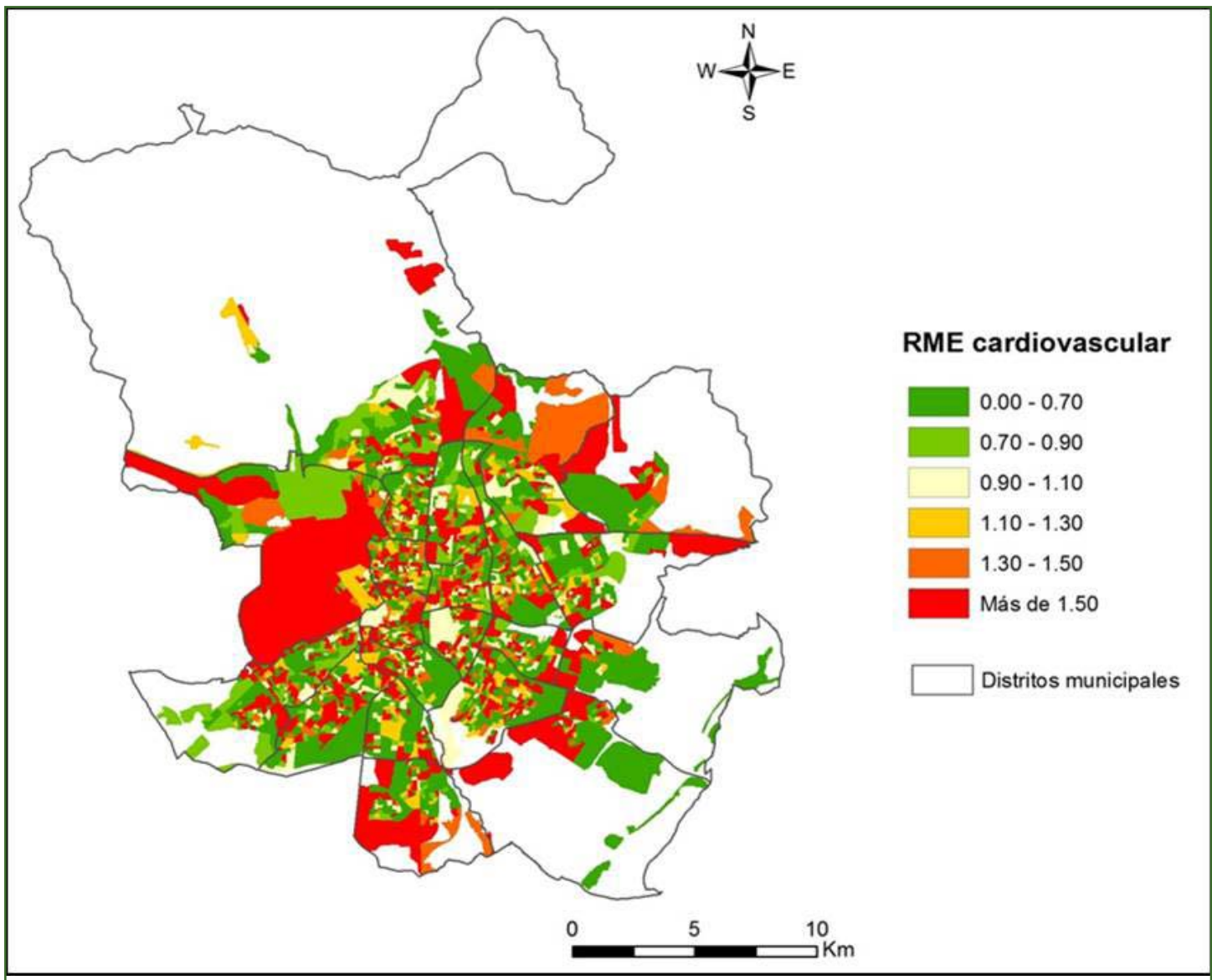

Figura 2. RME cardiovascular por secciones censales del AUP de Madrid 2010. Fuente: Elaboración propia. 


\section{Examinando posibles asociaciones entre contaminación por $\mathrm{PM}_{10}$ y RME cardio- vascular}

El análisis exploratorio bivariado de la relación entre las variables continuas de $\mathrm{PM}_{10}$ y RME cardiovascular nos muestra una correlación positiva, estadísticamente significativa, aunque débil (coeficiente de correlación de Spearman = 0,053, p-valor $=0,009$ ), sugiriendo que un aumento en la polución iría acompañado de un incremento en el riesgo cardiovascular. Por su parte, también se aprecia una asociación significativa, en la misma dirección, al aplicar la prueba U de Mann-Whitney, considerando la variable de RME de forma dicotómica, por encima y por debajo de la unidad, es decir, con un riesgo mayor al de la tasa del municipio o sin él (test = 673096, p-valor $=0,023$ ). La figura 3 ilustra esta relación mediante un gráfico de barras de error, donde se observa un valor promedio en los niveles $\mathrm{PM}_{10}$ relativamente superior en aquellas secciones censales con un mayor riesgo de mortalidad por enfermedad cardiovascular.

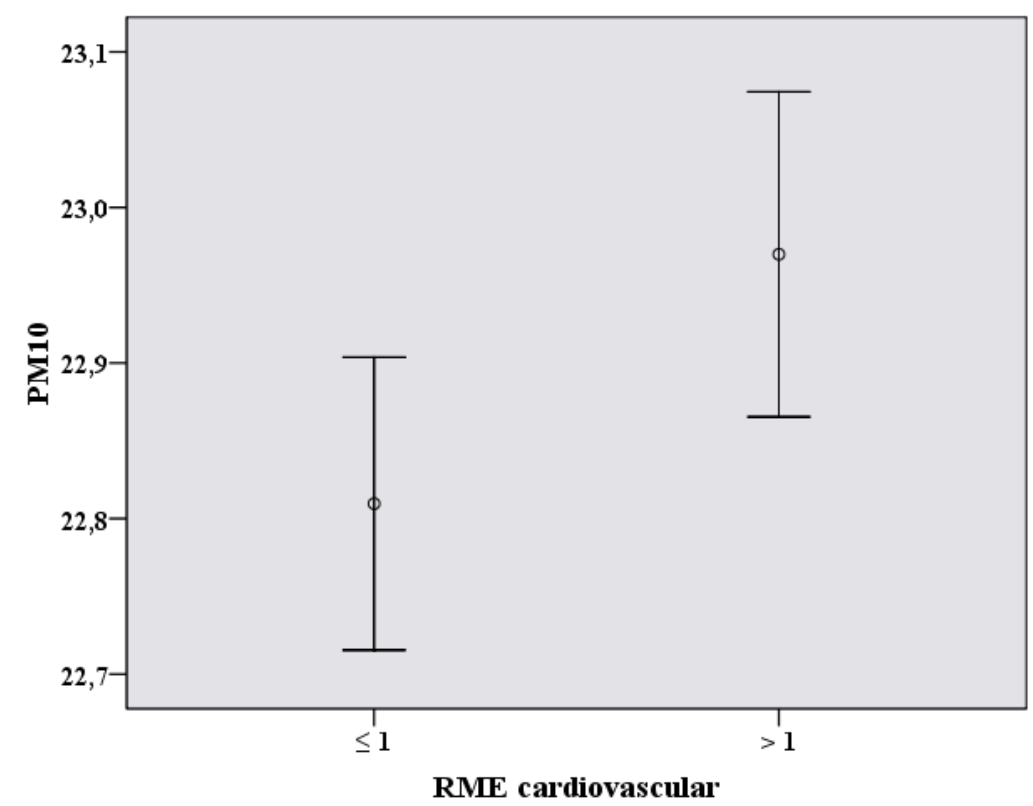

Figura 3. Barras de error de $\mathrm{PM}_{10}$ según nivel de RME cardiovascular para las secciones censales del AUP de Madrid, 2010.

Nota: El punto central de cada barra indica el valor medio de $\mathrm{PM}_{10}$ con respecto a $\mathrm{RME} \leq 1$ y $\mathrm{RME}>1$ y las barras los límites del intervalo de confianza al 95\%. Fuente: Elaboración propia.

La población total exhibe en Madrid una gama contrastada de valores, con algunas zonas más densas, sobre todo en algunos distritos del interior urbano (figura 4). En la franja más periférica las cifras suelen ser algo más bajas, si bien en la corona adyacente a ella, pero más interior (semi-periférica pues), suelen aparecer también cantidades algo destacadas en ciertos distritos del sur, del este o del norte. 


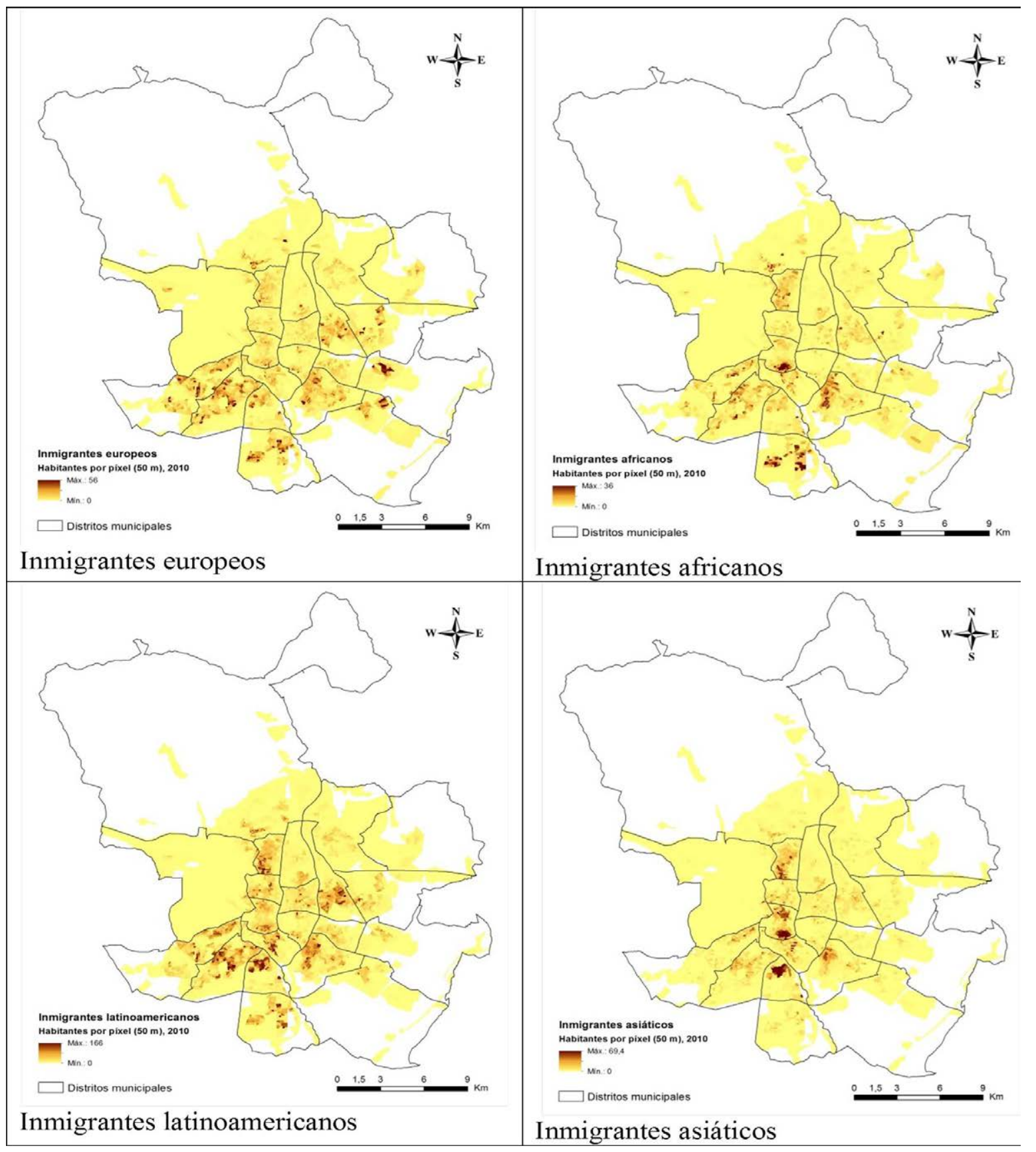

Figura 4. Distribución intraurbana de la población total y de tres grupos demográficos (mayores de 80 años y adultos según dos niveles de instrucción), Madrid, 2010-11.

Nota: Simbología con expansión del contraste mediante desviaciones estándar y parámetro de recorte $=6$.

Fuente: elaboración propia.

Desde el punto de vista de la edad, el patrón intraurbano de las personas de edad avanzada se ajusta a la forma habitualmente observada en las ciudades, apreciándose una presencia mayor en varios distritos interiores y en algunas zonas 
semi-periféricas del norte, este y suroeste (figura 4). Los adultos (30-59 años) con niveles de estudios altos y bajos exhiben formas contrapuestas, pues mientras los primeros abundan claramente en los distritos de la llamada "almendra central" y, a veces, en algunos del norte más periféricos, los segundos tienden a ubicarse en la parte meridional, amén de en ciertas zonas del distrito Centro (barrio de Embajadores), así como de Tetuán y en algunos lugares de la periferia norte, pero ya con guarismos inferiores.

La distribución intraurbana de los grupos de inmigrantes extranjeros analizados muestra ciertas coincidencias mutuas, pero también disparidades (figura 5). Las primeras atañen a la concurrencia importante de varios de tales grupos en las mismas zonas de la ciudad, en tanto que las segundas apuntan a variantes singulares en su localización. Por ejemplo, los latinoamericanos exhiben una presencia notable en bastantes áreas, especialmente en distritos de la semiperiferia meridional, en uno del este (Ciudad Lineal) y otro en el interior-norte (Tetuán). Por su parte los asiáticos aparecen mucho más concentrados en tres distritos (Centro, Tetuán y Usera) y alguno más (Puente de Vallecas). Los europeos abundan en zonas semiperiféricas del sur y este, en tanto que los africanos lo están en varias partes meridionales y en dos interiores (Centro y Tetuán en el centro-norte). Para un análisis más pormenorizado de ello remitimos al estudio de Palacios y Vidal ${ }^{51}$, quienes desvelan además que, por este orden, los asiáticos y los africanos están más segregados dentro de la ciudad y presentan una propensión mayor a la centralidad. Los europeos y latinoamericanos lo están menos en ambos criterios.

\section{Medición de desigualdades de exposición humana a ambientes dis- tintos de polución y mortalidad, desde la perspectiva de la JA}

\section{La distribución de los grupos demográficos según niveles de contaminación y de RME: análisis global}

Los resultados descriptivos presentados han permitido identificar contrastes espaciales en la distribución del contaminante $\mathrm{PM}_{10}$, la RME cardiovascular y la población en el municipio de Madrid. Es ahora el momento de comprobar si los distintos grupos sociodemográficos de población tienen una distribución similar a la de la población total en relación con el patrón espacial de $\mathrm{PM}_{10}$ Y RME, o si por el contrario, existen diferencias que sugieran una posible situación de inequidad.

El cuadro 5 exhibe cuatro grupos distintos de secciones censales del municipio de Madrid, clasificados en función del grado de contaminación por $\mathrm{PM}_{10}$ Y de la RME cardiovascular. Para estas cuatro categorías se recoge la población total y la de los subgrupos que habitan en zonas con bajo $\mathrm{PM}_{10}$ Y baja RME (situación más favorable), bajo $\mathrm{PM}_{10} \mathrm{y}$ alta RME, alto $\mathrm{PM}_{10} \mathrm{y}$ baja RME y, finalmente, alto $\mathrm{PM}_{10} \mathrm{Y}$ alta RME (situación más desfavorable).

51 Palacios García y Vidal Domínguez 2014 


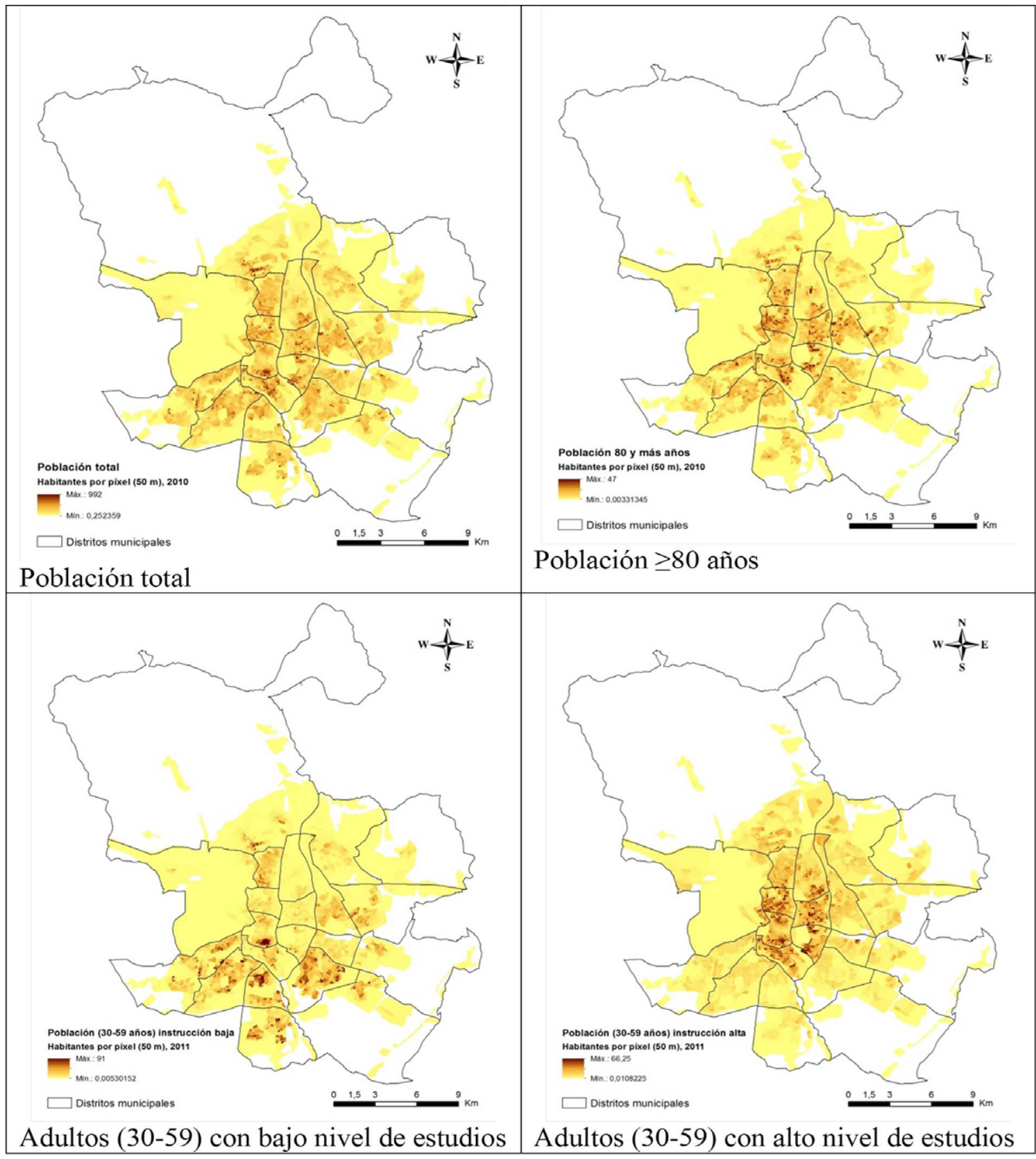

Figura 5. Distribución intraurbana de cuatro grupos demográficos de inmigrantes, Madrid, 2010. Nota: Simbología con expansión del contraste mediante desviaciones estándar y parámetro de recorte $=6$.

Fuente: elaboración propia.

En primer lugar, se aprecia que, de la confrontación de los porcentajes de cada uno de los grupos de población respecto a los de la población total, emergen diferencias significativas entre las distribuciones respectivas, por cuanto todos los valores de la prueba de $\chi^{2}$ avalarían el rechazo de $\mathrm{H}_{0}$ con unos niveles de probabilidad elevados, según denotan los $p$-valores obtenidos. Esa conclusión general cabe matizarla además en varios sentidos, considerando los propios 
valores del estadístico aplicado: en dos grupos demográficos, los adultos de alto nivel de instrucción y las personas de más de 80 años, la divergencia con respecto a la ciudad es especialmente notoria, a tenor de los muy altos valores del estadístico. En un segundo nivel cabría situar al grupo de inmigrantes europeos y en un tercer escalón estarían los asiáticos, los de bajo nivel de estudios y los africanos. Los latinoamericanos son el grupo que menos diverge de la distribución general observada en la ciudad. Podría interpretarse que, entre los inmigrantes, la mayor afinidad lingüístico-cultural de estos últimos con la población autóctona, propiciaría un reparto intraurbano más similar a ella, y por ende una exposición más parecida a situaciones ambientales y de mortalidad cardiovascular.

En segundo lugar, y para complementar la información e interpretación aportada por esa primera tabla, se presentan los resultados obtenidos mediante la prueba de diferencias entre proporciones, basada en el estadístico de contraste Z (cuadro 6), que permite identificar las categorías específicas que son más responsables de las diferencias significativas encontradas en el cuadro 5.

\begin{tabular}{|c|c|c|c|c|c|c|c|c|c|c|}
\hline & \multirow[b]{4}{*}{ Totales } & \multirow{2}{*}{\multicolumn{4}{|c|}{$\begin{array}{c}\mathrm{PM}_{10}<20 \mu \mathrm{g} / \mathrm{m}^{3} \\
\mathrm{RME} \text { cardiovascular }\end{array}$}} & \multirow{2}{*}{\multicolumn{4}{|c|}{$\begin{array}{c}\mathrm{PM}_{10} \geq 20 \mu \mathrm{g} / \mathrm{m}^{3} \\
\mathrm{RME} \text { cardiovascular }\end{array}$}} & \multirow{6}{*}{$\chi^{2}$} \\
\hline & & & & & & & & & & \\
\hline & & \multicolumn{2}{|c|}{$\leq 1$} & \multicolumn{2}{|c|}{$>1$} & \multicolumn{2}{|c|}{$\leq 1$} & \multicolumn{2}{|c|}{$>1$} & \\
\hline & & Total & $\%$ & Total & $\%$ & Total & $\%$ & Total & $\%$ & \\
\hline Secciones del AUP & 2400 & 102 & 4,25 & 81 & 3,38 & 1229 & 51,21 & 988 & 41,17 & \\
\hline Población total & 3272921 & 147918 & 4,52 & 126648 & 3,87 & 1628587 & 49,76 & 1369768 & 41,85 & \\
\hline Población 80 y más & 189250 & 5934 & 3,14 & 6749 & 3,57 & 88378 & 46,70 & 88189 & 46,60 & 2222.47 \\
\hline Bajo nivel de estudios & 166845 & 6027 & 3,61 & 5802 & 3,48 & 83800 & 50,23 & 71216 & 42,68 & 404.99 \\
\hline Alto nivel de estudios & 502443 & 30184 & 6,01 & 23461 & 4,67 & 245607 & 48,88 & 203191 & 40,44 & 3608.79 \\
\hline Europeos & 95347 & 4394 & 4,61 & 5110 & 5,36 & 48745 & 51,12 & 37098 & 38,91 & 781.57 \\
\hline Africanos & 42585 & 1285 & 3,02 & 1407 & 3,30 & 22714 & 53,34 & 17179 & 40,34 & 380.60 \\
\hline Latinoamericanos & 302098 & 12532 & 4,15 & 11759 & 3,89 & 149593 & 49,52 & 128214 & 42,44 & 121.11 \\
\hline Asiáticos & 51939 & 1776 & 3,42 & 1262 & 2,43 & 26112 & 50,27 & 22789 & 43,88 & 470.97 \\
\hline
\end{tabular}

Cuadro 5. Distribución de la población por áreas clasificadas según niveles de contaminación atmosférica por $\mathrm{PM}_{10}$ y razón estandarizada de mortalidad cardiovascular, Madrid, 2010.

Nota: La población total constituye el universo de referencia para las pruebas de $\chi^{2}$. $\mathrm{PM}_{10}<20 \mu \mathrm{g} / \mathrm{m}^{3}$ (valor medio anual fijado como límite por OMS). RME $>1$ : Riesgo mayor de mortalidad cardiovascular. En todos los casos el $p$-valor de $\chi^{2}$ fue inferior a 0,001.

Fuente: Elaboración propia.

El cuadro 6 nos permite detectar a simple vista aquellos grupos sociodemográficos cuyas proporciones son significativamente distintas a las de la población general en alguna de las cuatro categorías de estudio. Recuérdese que valores $>|2,58|$ serían significativos al nivel de $p=0,01$ en una prueba de dos colas. En conjunto solo tres proporciones no alcanzan dicho valor crítico, pero el resto avalarían tales divergencias. 


\begin{tabular}{|l|r|r|r|r|}
\hline & \multicolumn{2}{|c|}{$\mathbf{P M}_{\mathbf{1 0}}<\mathbf{2 0} \boldsymbol{\mu} \mathbf{g} / \mathbf{m}^{\mathbf{3}}$} & \multicolumn{2}{|c|}{$\mathbf{P M}_{\mathbf{1 0}} \geq \mathbf{2 0} \boldsymbol{\mu \mathbf { g } / \mathbf { m } ^ { \mathbf { 3 } }}$} \\
\hline & \multicolumn{2}{|c|}{$\mathrm{RME}$ cardiovascular } & \multicolumn{2}{c|}{$\mathrm{RME}$ cardiovascular } \\
\hline & \multicolumn{1}{|c|}{$\leq 1$} & \multicolumn{1}{|c|}{$\leq 1$} & $>1$ \\
\hline Población 80 y más & $-28,98$ & $-6,84$ & $-26,63$ & 41,87 \\
\hline Bajo nivel de estudios & $-17,84$ & $-8,30$ & 3,81 & 6,89 \\
\hline Alto nivel de estudios & 50,77 & 29,39 & $-12,43$ & $-20,27$ \\
\hline Europeos & 1,32 & 23,85 & 8,43 & $-18,42$ \\
\hline Africanos & $-14,92$ & $-6,05$ & 14,77 & $-6,32$ \\
\hline Latinoamericanos & $-9,82$ & 0,65 & $-2,65$ & 6,57 \\
\hline Asiáticos & $-12,07$ & $-17,01$ & 2,35 & 9,35 \\
\hline
\end{tabular}

Cuadro 6. Estadísticos de contraste Z para diferencias entre las proporciones de cada uno de los grupos de población y a las de la población total de Madrid, 2010.

Nota: Se trata de valores estandarizados del estadístico, que indican la distancia respecto a la situación en la población general (valor 0). Resaltada celdas con Z > 8 .

Fuente: Elaboración propia.

El examen de la columna primera (izqda.) resalta, como caso notable por su alto valor de Z, al grupo de personas con un elevado nivel de estudios, pues se encuentra altamente sobrerrepresentado en aquellas secciones censales con una baja contaminación ambiental por $\mathrm{PM}_{10}$ y un bajo riesgo de mortalidad cardiovascular. Ello expresa la divergencia más acusada respecto a la población de la ciudad, destacando además que implica vivir proporcionalmente más en áreas privilegiadas en contaminación (baja) y mortalidad cardiovascular (baja). Recordemos que el porcentaje de población total que habita en esta zona es de 4,52 \%, mientras que el de este grupo sociodemográfico es de 6,01\%, como puede verse en el cuadro 5. Por el contrario, resulta revelador que en esta zona la mayoría de los grupos restantes (vulnerables) se hallan infrarrepresentados en mayor o menor grado, tal como se desprende del hecho de que los valores de $\mathrm{Z}$ sean negativos y algunos bastante negativos.

De forma opuesta, la columna extrema derecha define la situación más negati$\mathrm{va}$, es decir, las secciones censales más desfavorecidas en cuanto a polución por $\mathrm{PM}_{10}$ y mortalidad cardiovascular. Allí destaca la proporción de la población de 80 y más años de edad por su importante presencia (Cuadro 6). Si el porcentaje de población general constituye un $41,85 \%$ en este ámbito, las personas mayores alcanzan un 46,60 \% (cuadro 5), denotando pues una situación más desfavorable. Aunque con valores Z más bajos (pero positivos) los asiáticos y, en menor grado, los latinoamericanos y los adultos de bajo nivel de instrucción aparecen como algo desfavorecidos. En el cuadro 5 todos esos grupos superan en sus porcentajes al global de la ciudad en esa categoría. Por el contrario, los adultos de alto nivel de instrucción y los inmigrantes europeos están infrarrepresentados, i.e. en situación algo menos perjudicial.

Las condiciones que caracterizan a las dos columnas centrales parecen menos claras de interpretar en una primera mirada. Corresponden a dos tipos de entornos: a) zonas donde las $\mathrm{PM}_{10}$ son bajas, pero la RME es alta (segunda columna) en las 
que aparecen sobrerrepresentados los adultos de alta instrucción y los inmigrantes europeos ( $\mathrm{Z}$ altas y positivas); $\mathrm{y}$ b) zonas con altas $\mathrm{PM}_{10^{\prime}}$ pero baja RME (tercera columna) en las que destaca la presencia de inmigrantes africanos y algo de los europeos ( $\mathrm{Z}$ moderadas y positivas). Al respecto conviene recordar aquí que la relación espacial entre polución de $\mathrm{PM}_{10}$ y RME cardiovascular puede no ser necesariamente nítida, pues la trasposición al espacio de esa hipotética causalidad está muy supeditada a la agregación de los datos y a su forma de espacialización, como en algún estudio análogo se ha comprobado ${ }^{52}$. La búsqueda de concomitancias espaciales entre ambos fenómenos y la presencia de ciertos grupos demográficos, a la hora de su interpretación racional, no puede soslayar el hecho de que esas relaciones son complejas y que sería poco plausible asumir que inmigrantes con corta estadía en Madrid estuviesen incursos en mortalidad cardiovascular por causa de las $\mathrm{PM}_{10}$. Tras esas consideraciones cabría añadir algo sobre las coincidencias recién señaladas para los grupos poblacionales: los adultos de alta instrucción son consistentes en su prevalencia en zonas de bajas $\mathrm{PM}_{10^{\prime}}$ sea cual fuere la RME, aunque abundan más en zonas de baja RME, como se señaló antes. Los africanos abundan proporcionalmente algo más solo en zonas de altas $\mathrm{PM}_{10^{\prime}}$ resultado de su concentrada localización, pero de baja RME cardiovascular (causa de la que presumiblemente fallezcan pocos, por su composición de edad). Los inmigrantes europeos, finalmente, exhiben una sobrerrepresentación sobre todo en zonas de baja polución y alta RME (y un poco en zonas de rasgos opuestos a los anteriores), por lo que la interpretación de ello resulta algo arriesgada.

\section{Identificando asociaciones espaciales entre polución, mortalidad y grupos demo- gráficos a través de la visualización cartográfica}

Junto a los resultados de los test estadísticos, el recurso a la cartografía permite patentizar de una forma importante, esto es visualmente, las asociaciones entre las distribuciones espaciales que estamos tratando de poner de manifiesto. Ello es posible mediante las herramientas disponibles en SIG, que facilitan la exploración interactiva y simultánea de los tres tipos de fenómenos considerados (polución, mortalidad cardiovascular y grupos sociodemográficos), mediante búsquedas selectivas que muestren los patrones espaciales combinados.

Un ejemplo de ello se presenta en la figura 6. Su objetivo ha sido ilustrar el caso de los dos grupos de población cuya divergencia respecto al total de la ciudad se ha mostrado más intensa: los de 80 años y más y los adultos con alto nivel de instrucción. En relación a los primeros, el resultado que en el mapa se muestra en tono rojizo identifica las secciones donde converge una alta contaminación $\left(\mathrm{PM}_{10}>20 \mu \mathrm{g} /\right.$ $\mathrm{m}^{3}$ ), una RME desfavorable, por alta (>1), y una densidad de personas de edad avanzada superior a la mediana de las secciones censales. Se trata pues de una situación en la que abundan relativamente los adultos mayores y que ostenta rasgos negati-

52 Portnov, Dubnov y Barchana 2009 
vos en contaminación y en mortalidad cardiovascular. Las zonas que cumplen esos criterios predominan en los distritos del interior de la ciudad (barrios del Ensanche) y en la periferia más cercana, los cuales se urbanizaron en el XIX y primera mitad del XX y por tanto están consolidadas y con claro envejecimiento.

El grupo de los adultos con instrucción superior se ha explorado espacialmente imponiendo las restricciones de baja contaminación $\left(\mathrm{PM}_{10}<20 \mu \mathrm{g} / \mathrm{m}^{3}\right)$ y baja RME cardiovascular $(<1)$, junto con densidad elevada (>mediana) de dichos adultos. Se trata, como se vio en el apartado anterior, del caso y grupo más favorecido. La cifra de tales secciones es mucho más exigua en la ciudad y su ubicación, denotada en el mapa de la figura 6 con color azul, aparece en solo unos pocos lugares de la metrópoli: el norte, el oeste y el suroeste. Debe recordarse al respecto que la contaminación por $\mathrm{PM}_{10}$ era baja en esa banda periférica y que grupos socioeconómicos medio-altos y altos aparecen en zonas concretas de la periferia urbana de Madrid (en edificios residenciales multifamiliares de cierta densidad y de buena calidad). Estas coincidencias espaciales, muy sintomáticas, son las que subyacen y avalan a los resultados alcanzados en los test estadísticos previamente expuestos.

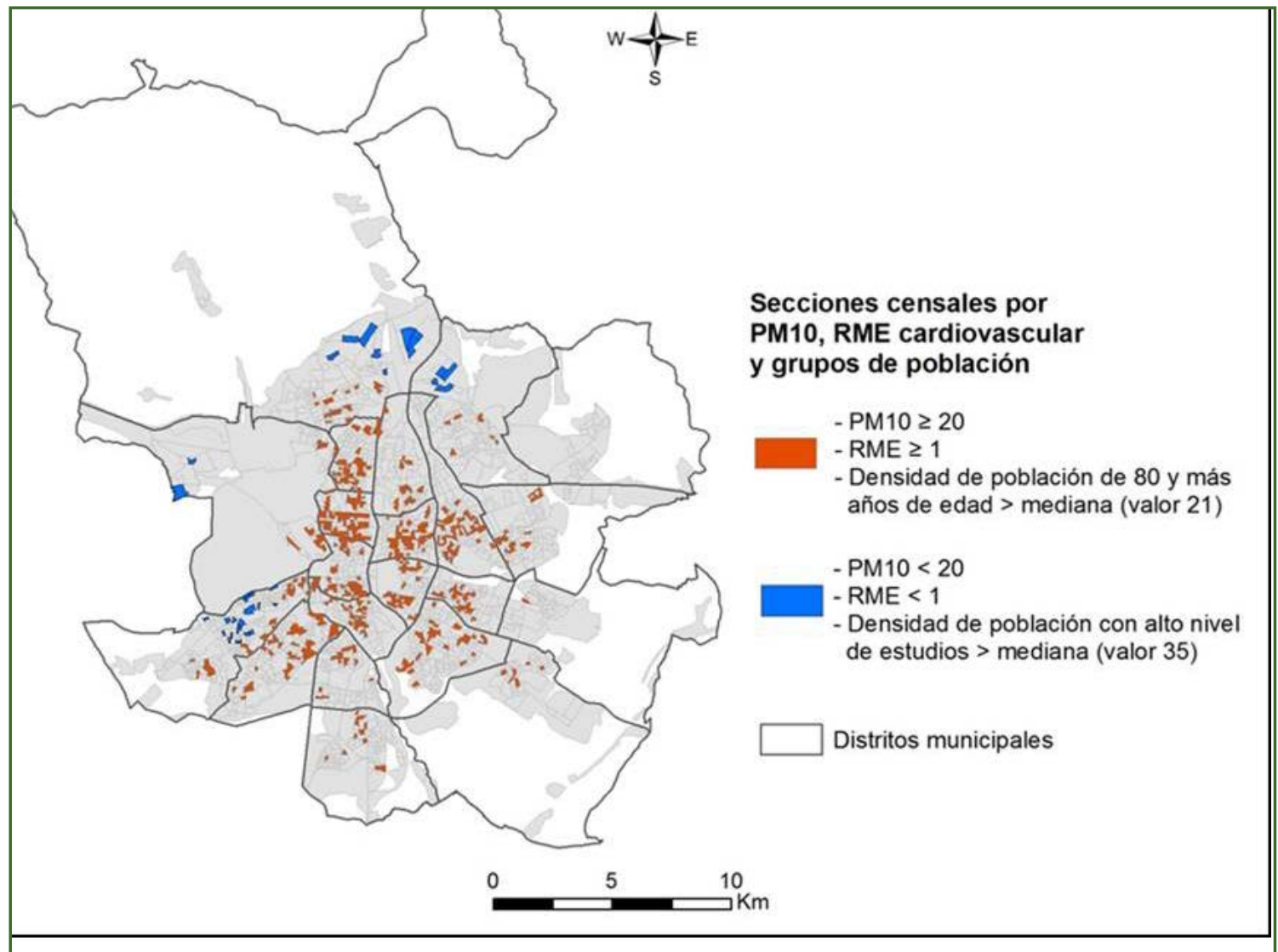

Figura 6. Mapa de las secciones con importante densidad de personas de edad avanzada (en rojo) o de adultos de alta instrucción (en azul) que cumplen ciertos criterios favorables y desfavorables en polución y mortalidad cardiovascular.

Fuente: Elaboración propia 


\section{Discusión y conclusiones}

En este trabajo se ha ofrecido una aproximación, desde la perspectiva de la justicia ambiental, al estudio de la posible interacción entre la contaminación atmosférica, la mortalidad cardiovascular y ciertos grupos vulnerables de población en la ciudad de Madrid.

Metodológicamente, se aplicaron diversas técnicas estadísticas y de SIG que permitieron combinar información procedente de diferentes bases de datos y analizar de forma integrada variables correspondientes a la triple dimensión ambiental, social y de la salud. El área de estudio fue el AUP, con su desagregación a nivel de sección censal, con el fin de acotar los análisis a aquellas zonas del municipio de Madrid con presencia habitual de residentes. La capa de $\mathrm{PM}_{10}$ se obtuvo mediante interpolación, siguiendo el modelo de Kriging anisotrópico, a partir de los datos registrados en cada estación de medición de calidad del aire de la ciudad de Madrid. Con relación a la dimensión social, se incluyó a la población residente en cada sección censal y se definieron grupos sociodemográficos de acuerdo con el nivel de estudios, la edad (de 80 años y más años) y el ámbito de origen de los habitantes procedentes de países menos desarrollados. La mortalidad por enfermedades cardiovasculares se midió a través de la RME ajustada por edad, calculando los casos esperados de cada sección censal y tomando como referencia la tasa del municipio.

Trabajos previos en este campo han incluido el estudio conjunto de la polución atmosférica por partículas, la morbilidad y mortalidad por causas cardiovasculares, y variables socioeconómicas tales como desempleo, ingresos del hogar y escolarización ${ }^{20}$, segregación residencial socioeconómica y racial ${ }^{53}$, estatus socioeconómico ${ }^{54} \mathrm{y}$ edad avanzada ${ }^{55}$. Algunas de las técnicas de análisis aplicadas en ellos incluyen modelos estadísticos de regresión, en la búsqueda de relaciones entre las características socioeconómicas, la contaminación por partículas y la mortalidad cardiovascular. En el presente trabajo el énfasis ha recaído en conocer la distribución de distintos grupos sociodemográficos en zonas de mayor y menor contaminación por partículas de tamaño inferior o igual a $10 \mu \mathrm{m}$ y de mayor y menor riesgo relativo de mortalidad por enfermedades cardiovasculares, con el propósito de identificar posibles inequidades socioespaciales en el territorio.

Con respecto al área geográfica, se han demostrado las ventajas de utilizar una escala intraurbana y de gran nivel de detalle en este tipo de investigaciones ${ }^{8}$, pues aportan una información que a una escala metropolitana y con unidades mayores puede quedar más difuminada. La utilización en este estudio del AUP y de la sección censal como unidad espacial de análisis en la ciudad de Madrid sigue esa premisa.

Al estudiar el espacio urbano madrileño en función de la contaminación atmosférica y la mortalidad cardiovascular, los resultados de esta investigación sugieren,

53 Schulz et al. 2005

54 Gouveia y Fletcher 2000

55 Barnett et al. 2006 
en primer lugar, una asociación significativa entre ambas variables, de manera que en secciones con una concentración mayor de $\mathrm{PM}_{10}$ se observa una RME relativamente mayor.

En segundo lugar, es importante destacar que los distintos grupos analizados muestran diferencias significativas cuando se comparan con la distribución intraurbana de la población madrileña en su conjunto. Así, en las secciones censales que tienen un bajo nivel de polución y de RME, se encuentra representado en mayor proporción el grupo de personas con un nivel de estudios más elevado, mientras que los demás grupos están infrarrepresentados allí (personas con un bajo nivel de estudios, población de edad avanzada, europeos, africanos, latinoamericanos y asiáticos). La distribución espacial de las secciones más favorecidas corresponde a la franja periférica norte, oeste y suroeste, caracterizada por sus viviendas de clase media hasta alta.

Estudios anteriores evidencian una asociación directa entre la exposición a partículas en suspensión $\left(\mathrm{PM}_{10}\right.$ y $\left.\mathrm{PM}_{2,5}\right)$ y mortalidad por enfermedades cardiovasculares y respiratorias ${ }^{56}$. Así mismo, los resultados del presente trabajo sobre polución, salud y nivel de estudios, convergen con los de investigaciones previas como la de Schikowski et al. ${ }^{57}$, llevada a cabo en población femenina, en la que se definió el estatus socioeconómico mediante el nivel educativo y se demostró una relación significativa entre una mayor exposición a $\mathrm{PM}_{10} \mathrm{y}$ un bajo nivel educativo. A su vez, se observó una asociación entre este indicador socioeconómico y problemas de salud respiratoria que, como indican los autores, puede ser explicado en cierta medida por las condiciones de vida desfavorables de la población de estudio, entre las que se considera la contaminación atmosférica. Por su parte, los resultados de Wong et al. ${ }^{20}$ sugieren que los riesgos de mortalidad cardiovascular asociados a la contaminación del aire por $\mathrm{PM}_{10^{\prime}} \mathrm{SO}_{2}$ y $\mathrm{NO}_{2}$ pueden incrementarse en los barrios de menor nivel socioeconómico.

Además de los grupos de población por nivel de estudios, la edad también reveló diferencias significativas con relación al patrón de la población en su conjunto. Así, se observó que en las secciones censales con una mayor contaminación atmosférica por partículas $\mathrm{PM}_{10}$ y con un riesgo más elevado de mortalidad cardiovascular (controlando el efecto de la edad) reside, en una mayor proporción, la población de 80 y más años de edad. Corresponden a estas características ambientales, de salud y demográficas, secciones centrales del ensanche y secciones de la periferia más próxima al este y sur de Madrid, donde las viviendas son en general más modestas que en las zonas identificadas anteriormente.

Estudios como los desarrollados por Gouveia y Fletcher ${ }^{54}$ sugieren que la influencia de la polución del aire por $\mathrm{PM}_{10}$ en el riesgo de mortalidad cardiovascular aumenta en las personas mayores. Por su parte, Barnett et al. ${ }^{55}$ observaron asociaciones significativas entre un incremento en las concentraciones de contaminantes

56 Pelucchi et al. 2009

57 Schikowski et al. 2008 
como $\mathrm{NO}_{2}$ y $\mathrm{PM}_{10}$ y un aumento en los ingresos hospitalarios por enfermedades cardiovasculares en personas de edad avanzada.

Si bien los resultados expuestos en el presente estudio han permitido identificar una situación de inequidad en el patrón espacial de la contaminación del aire, la mortalidad cardiovascular y los grupos vulnerables, no es posible inferir simplistamente relaciones de causa y efecto a partir de los datos analizados. Sin embargo, cabe plantear las hipótesis sobre la exposición diferencial y la susceptibilidad diferencial de los distintos grupos de población ${ }^{7}$. Por un lado, en la ciudad de Madrid la exposición a los contaminantes no parece ser la misma para todos los habitantes, pues aquellos con un nivel de estudios más elevado residirían en mayor proporción en zonas con un bajo nivel de polución. Contrariamente, habría grupos más expuestos a la contaminación por residir en secciones censales con mayores concentraciones de $\mathrm{PM}_{10}$, como es el caso de las personas de edad avanzada. Por otro lado, determinados grupos de población serían más susceptibles que otros a los efectos de los contaminantes en su salud, lo que sumado a una mayor exposición a dichos contaminantes podría contribuir a explicar los patrones espaciales observados, como el correspondiente a las secciones donde converge una alta contaminación, una mayor densidad de población de 80 y más años de edad y un mayor riesgo de mortalidad cardiovascular.

En resumen, este estudio ha aportado un planteamiento metodológico original, desde la perspectiva de la justicia ambiental, para acometer el análisis conjunto de datos sobre facetas muy diversas, pero interactuantes, relativas a contaminación atmosférica, mortalidad cardiovascular y grupos vulnerables de población, que ha permitido determinar la existencia de inequidades espaciales con implicaciones ambientales, sociales y de salud. Ante este tipo de escenarios, crece la necesidad de seguir profundizando en esta línea y de aportar conocimiento válido para orientar políticas urbanas, social y ambientalmente justas. En futuros trabajos se prevé continuar con esta problemática, considerando otros contaminantes y otras causas de mortalidad.

\section{Bibliografía}

ARRIAGA LEGARDA, A. y PARDO BUENDÍA, M. Justicia ambiental. El estado de la cuestión. Revista Internacional de Sociología, 201 1, vol. 69, n 3, p. 627-648.

BARNETT, A.G., WILLIAMS, G.M., SCHWARTZ, J., BEST, T.L., NELLER, A.H., PETROESCHEVSKY, A.L. y SIMPSON, R.W. The effects of air pollution on hospitalizations for cardiovascular disease in elderly people in Australian and New Zealand cities. Environmental Health Perspectives, 2006, p. 1018-1023.

BOSQUE SENDRA, J., DÍAZ CASTILLO, C. y DÍAZ MUÑOZ, M.D.L.A. De la justicia espacial a la justicia ambiental en la política de localización de instalaciones para la gestión de residuos en la Comunidad de Madrid Boletín de la Real Sociedad Geográfica, 2001-2002, no 137-138, p. 89-114. 
BRAUER, M., HOEK, G., VAN VLIET, P., MELIEFSTE, K., FISCHER, P., GEHRING, U., HEINRICH, J., CYRYS, J., BELLANDER, T. y LEWNE, M. Estimating long-term average particulate air pollution concentrations: application of traffic indicators and geographic information systems. Epidemiology, 2003, vol. 14, nº 2, p. 228-239.

BRULLE, R.J. y PELLOW, D.N. Environmental justice: human health and environmental inequalities. Annual Review of Public Health, 2006, vol. 27, p. 103-124.

BULLARD, R.D. Growing smarter: achieving livable communities, environmental justice, and regional equity. Cambridge: MIT Press, 2007.

BUZZELLI, M. y JERRETT, M. Racial gradients of ambient air pollution exposure in Hamilton, Canada. Environment and Planning A, 2004, vol. 36, n 10, p. 1855-1876.

CAÑADA TORRECILLA, M.R. Análisis Geoestadístico. In A. MORENO JIMÉNEZ ed. Sistemas y análisis de la información geográfica. Madrid: Ra-Ma, 2007, p. 755-880.

CAÑADA TORRECILLA, M.R., MORENO JIMÉNEZ, A. y GONZÁLEZ LORENZO, H. Modelado de la calidad del aire urbano. Un experimento metodológico con técnicas de interpolación espacial. Boletín de la Asociación de Geógrafos Españoles, 2014, nº 65, p. 317-342.

CAÑADA TORRECILLA, R., VIDAL DOMÍNGUEZ, M.J. y MORENO JIMÉNEZ, A. Interpolación espacial y visualización cartográfica para el análisis de la justicia ambiental: ensayo metodológico sobre la contaminación por partículas atmosféricas en Madrid. GeoFocus, 2011 , n 11, p. 118-154.

DALY, A. y ZANNETTI, P. Air pollution modeling: an overview. In P. ZANNETTI, D. AL-AJMI y S. AL-RASHIED eds. Ambient air pollution. Fremont: The Arab School for Science and Technology (ASST) and The Enviro. Comp. Institute, 2007.

DE MESNARD, L. Pollution models and inverse distance weighting: Some critical remarks. Computers \& Geosciences, 2013, vol. 52, p. 459-469.

DEGUEN, S. y ZMIROU-NAVIER, D. Social inequalities resulting from health risks related to ambient air quality - a European review. The European Journal of Public Health, 2010, vol. 20, nº 1, p. 27-35.

DOCKERY, D.W. Epidemiologic evidence of cardiovascular effects of particulate air pollution. Environmental health perspectives, 2001, vol. 109, nº Suppl 4, p. 483.

FAN, X., LAM, K.-C. y YU, Q. Differential exposure of the urban population to vehicular air pollution in Hong Kong. Science of the Total Environment, 2012, vol. 426, p. 211-219.

FUNDACIÓN MAPFRE. Salud y sostenibilidad: efectos de la calidad del aire urbano. Madrid: Fundación Mapfre. Instituto de Instituto de Prevención Salud y Medio Ambiente. Observatorio de la Sostenibilidad en España, 2013.

GÓMEZ-BARROSO, D., PRIETO-FLORES, M.E., MELLADO SAN GABINO, A. y MORENO JIMÉNEZ, A. Análisis espacial de la mortalidad por enfermedades cardiovasculares en la ciudad de Madrid, España. Revista Española de Salud Pública, 2015, vol. 89, n ${ }^{\circ} 1$, p. 27-37.

GOUVEIA, N. y FLETCHER, T. Time series analysis of air pollution and mortality: effects by cause, age and socioeconomic status. Journal of epidemiology and community 
health, 2000, vol. 54, no 10, p. 750-755.

GURJAR, B.R., MOLINA, L.T. y OJHA, C.S.P. Air pollution: health and environmental impacts. CRC Press. Taylor and Francis, 2010.

HORTELANO, J.A. Estadística para psicólogos II: Probabilidad. Estadistica inferencial. Madrid: Pirámide, 2000.

INSTITUTE OF MEDICINE COMMITTEE ON ENVIRONMENTAL JUSTICE. Toward environmental justice: Research, education, and health policy needs. National Academies Press (US), 1999.

JERRETT, M., BURNETT, R.T., KANAROGLOU, P., EYLES, J., FINKELSTEIN, N., GIOVIS, C. y BROOK, J.R. A GIS-environmental justice analysis of particulate air pollution in Hamilton, Canada. Environment and Planning A, 2001, vol. 33, n 6, p. 955-974.

JERRETT, M., BURNETT, R.T., MA, R., POPE, C.A., KREWSKI, D., NEWBOLD, K.B., THURSTON, G., SHI, Y., FINKELSTEIN, N., CALLE, E.E. y THUN, M.J. Spatial Analysis of Air Pollution and Mortality in Los Angeles. Epidemiology, 2005, vol. 16, nº 6, p. 727-736.

KRIVORUCHKO, K. Spatial statistical data analysis for GIS users. Redlands, CA: Esri Press, 2011.

LANDRIGAN, P.J., RAUH, V.A. y GALVEZ, M.P. Environmental justice and the health of children. The Mount Sinai Journal of Medicine, 2010, vol. 77, nº 2, p. 178-187.

LI, J. y HEAP, A.D. A review of comparative studies of spatial interpolation methods in environmental sciences: performance and impact factors. Ecological Informatics, 201 1, vol. 6, no 3, p. 228-241.

MAANTAY, J. Mapping environmental injustices: pitfalls and potential of geographic information systems in assessing environmental health and equity. Environmental Health Perspectives, 2002, vol. 110, no Suppl 2, p. 161.

MAESO MARTÍNEZ, S.,ZORRILLATORRAS, B., GALÁN LABACA,I., GANDARILLAS GRANDE, A.M. y CANTERO, J.L. Situación epidemiológica de las enfermedades cardiovasculares en la Comunidad de Madrid, 2005. Boletín Epidemiológico de la Comunidad de Madrid, 2006, vol. 12, nº 4.

MAROKO, A.R. Using air dispersion modeling and proximity analysis to assess chronic exposure to fine particulate matter and environmental justice in New York City. Applied Geography, 2012, vol. 34, p. 533-547.

MOHAI, P., PELLOW, D. y ROBERTS, J.T. Environmental justice. Annual Review of Environment and Resources, 2009, vol. 34, p. 405-430.

MORENO JIMÉNEZ, A. ¿Está equitativamente repartida la contaminación sonora urbana? Una evaluación desde el principio de justicia ambiental en la ciudad de Madrid. Estudios Geográficos, 2007, vol. 68, no 263, p. 595-626.

MORENO JIMÉNEZ, A. Justicia Ambiental. Del concepto a la aplicación en análisis de políticas y planificación territoriales. Scripta Nova, 2010, vol. XIV, no 316.

MORENO JIMÉNEZ, A. Evaluación de la justicia ambiental con sistemas de información geográfica. In A. MORENO-JIMÉNEZ, BUZAI, G. D. Y FUENZALIDA-DÍAZ, 
M. ed. Sistemas de información geográfica. Aplicaciones en diagnósticos territoriales y decisiones geoambientales. Madrid: Ra-Ma, 2012, p. 173-202.

MORENO JIMÉNEZ, A. Población y polución atmosférica intraurbana por dióxido de nitrógeno en Madrid: análisis desde la justicia ambiental basado en Sistemas de Información Geográfica. Cuadernos geográficos, 2013, vol. 52, nº 1, p. 84-107.

MORENO JIMÉNEZ, A., CAÑADA TORRECILLA, R., VIDAL DOMÍNGUEZ, M.J., PALACIOS GARCÍA, A. Y MARTÍNEZ SUÁREZ, P. Assessing environmental justice through potential exposure to air pollution: A socio-spatial analysis in Madrid and Barcelona, Spain. Geoforum, 2016, vol. 69, p. 117-131.

PALACIOS GARCÍA, A. y VIDAL DOMÍNGUEZ, M.J. La distribución intraurbana de los inmigrantes en las ciudades españolas: un análisis de casos con SIG y técnicas cuantitativas. Cuadernos Geográficos, 2014, vol. 53, nº 1, p. 98-121.

PELUCCHI, C., NEGRI, E., GALLUS, S., BOFFETTA, P., TRAMACERE, I. y LA VECCHIA, C. Long-term particulate matter exposure and mortality: a review of European epidemiological studies. BMC public health, 2009, vol. 9, nº 1, p. 453.

PORTNOV, B.A., DUBNOV, J. y BARCHANA, M. Studying the association between air pollution and lung cancer incidence in a large metropolitan area using a kernel density function. Socio-Economic Planning Sciences, 2009, vol. 43, no 3, p. 141-150.

PYE, S., STEDMAN, J., ADAMS, M. Y KING, K. Further analysis of $\mathrm{NO}_{2}$ and $\mathrm{PM}_{10}$ air pollution and social deprivation. Abingdon: AEA Technology, 2001.

QUEROL, X., AlASTUEY, A., MORENO, T., VIANA, M.M., CASTILlO, S., PEY, J., RODRÍGUEZ, S., CRISTÓBAL, A., JIMÉNEZ, S., PALLARÉS, M., DE LA ROSA, J., ARTIÑANO, B., SALVADOR, P., SÁNCHEZ, M., GARCÍA, S., HERCE, M.D., FERNÁNDEZPATIER, R., MORENO-GRAU, S., NEGRAL, L., MINGUILLÓN, M.C., MONFORT, E., SANZ, M.J., PALOMO-MARÍN, R., PINILLA_GILL, E. y CUEVAS, E. Material particulado en España: niveles, composición y contribución de fuentes. Madrid: CSIC y Ministerio de Medio Ambiente, 2006.

QUEROL, X., ALASTUEY, A., RODRÍGUEZ, S., VIANA, M.M., ARTIÑANO, B., SALVADOR, P., MANTILLA, E., SANTOS, S.G.D., PATIER, R.F., ROSA, J.D.L., CAMPA, A.S.D.L. y MENEDEZ, M. Estudio y evaluación de la contaminación atmosférica por material particulado en España:Informes finales. IJA-CSIC, ISCIII, CIEMAT, Universidad de Huelva, Universidad del País Vasco, 2003.

RODRÍGUEZ, S., QUEROL, X., ALASTUEY, A. y PLANA, F. Sources and processes affecting levels and composition of atmospheric aerosol in the Western Mediterranean. Journal of Geophysical Research, 2002, vol. 107, nº D24, p. AAC 12-11AAC 12-14.

ROUTLEDGE, H.C. Y AYRES, J.G. Air pollution and the heart. Occupational Medicine, 2005, vol. 55, nº 6, p. 439-447.

RUIZ-MAYA, L., MARTÍN PLIEGO, F.J., MONTERO, J.M. y URIZ TOMÉ, P. (eds.) Análisis estadístico de encuestas: datos cualitativos. Madrid: A.C, 1995.

SCHIKOWSKI, T., SUGIRI, D., REIMANN, V., PESCH, B., RANFT, U. Y KRÃMER, U. Contribution of smoking and air pollution exposure in urban areas to social 
differences in respiratory health. BMC public health, 2008, vol. 8, $\mathrm{n}^{\circ}$ 1, p. 179.

SCHULZ, A.J., KANNAN, S., DVONCH, J.T., ISRAEL, B.A., ALLEN III, A., JAMES, S.A., HOUSE, J.S. y LEPKOWSKI, J. Social and physical environments and disparities in risk for cardiovascular disease: the healthy environments partnership conceptual model. Environmental Health Perspectives, 2005, vol. 113, no 12, p. 1817-1825.

SIEGEL, S. (ed.) Estadística no paramétrica aplicada a las ciencias de la conducta. México: Trillas, 1972.

SU, J.G., LARSON, T., GOULD, T., COHEN, M. y BUZZELLI, M. Transboundary air pollution and environmental justice: Vancouver and Seattle compared. GeoJournal, 2010, vol. 75, nº 6, p. 595-608.

VIANA, M., PÉREZ, C., QUEROL, X., ALASTUEY, A., NICKOVIC, S. y BALDASANO, J.M. Spatial and temporal variability of PM levels and composition in a complex summer atmospheric scenario in Barcelona (NE Spain). Atmospheric Environment, 2005, vol. 39, nº 29, p. 5343-5361.

VIDAL DOMÍNGUEZ, M.J. y PALACIOS GARCÍA, A. Apuntes metodológicos para el estudio de las desigualdades sociodemográficas intraurbanas. In P. REQUES y O. COS eds. La población en clave territorial. Procesos, estructuras y perspectivas. XIII Congreso de Población Española. Santander: Universidad de Cantabria, 2012, p. 457464.

WALKER, G. Environmental justice: concepts, evidence and politics. New York: Routledge, 2012.

WALKER, G.P. y BULKELEY, H. Geographies of Environmental Justice. Geoforum, 2006, vol. 37, no 5, p. 655-659.

WANG, G., VAN DEN BOSCH, F.H.M. y KUFFER, M. Modelling urban traffic air pollution dispersion. . In ISPRS 2008: Proceedings of the XXI Congress: Silk road for information from imagery. The International Society for Photogrammetry and Remote Sensing. Beijing, China, 2008, p. 153-158.

WONG, C.M., OU, C.Q., CHAN, K.P., CHAU, Y.K., THACH, T.Q., YANG, L., CHUNG, R.Y.-N., THOMAS, G.N., PEIRIS, J.S.M. y WONG, T.-W. The effects of air pollution on mortality in socially deprived urban areas in Hong Kong, China. Environmental Health Perspectives, 2008, vol. 116, nº 9, p. 1189-1194.

WONG, D.W., YUAN, L. y PERLIN, S.A. Comparison of spatial interpolation methods for the estimation of air quality data. Journal of Exposure Science and Environmental Epidemiology, 2004, vol. 14, nº 5, p. 404-415.

WORLD HEALTH ORGANIZATION. Environment and health risks: a review of the influence and effects of social inequalities. Copenhagen: WHO, Regional Office for Europe, 2010.262 p.

WORLD HEALTH ORGANIZATION. Review of evidence on health aspects of air pollution-REVIHAAP Project. Copenhagen: 2013. 
(c) Copyright: María-Eugenia Prieto-Flores, Antonio Moreno Jiménez, Diana Gómez-Barroso, Rosa Cañada Torrecilla, Rosa y Pedro Martínez Suárez, , 2017.

(c) Copyright Scripta Nova, 2017.

Ficha bibliográfica:

PRIETO-FLORES, María-Eugenia; MORENO JIMÉNEZ, Antonio; GÓMEZ-BARROSO, Diana; CAÑADA TORRECILLA, Rosa; MARTINEZ SUÁREZ, Pedro. Contaminación del aire, mortalidad cardiovascular y grupos vulnerables en Madrid: un estudio exploratorio desde la perspectiva de la justicia ambiental. Scripta Nova. Revista Electrónica de Geografía y Ciencias Sociales. [En línea]. Barcelona: Universidad de Barcelona, 1 de marzo de 2017, vol. XXI, no 559. [ISSN: 1138-9788]. 